\title{
Sequential Modular Position and Momentum Measurements of a Trapped Ion Mechanical Oscillator
}

\author{
C. Flühmann, ${ }^{*}$ V. Negnevitsky, M. Marinelli, and J. P. Home ${ }^{\dagger}$ \\ Institute for Quantum Electronics, ETH Zürich, Otto-Stern-Weg 1, 8093 Zürich, Switzerland
}

(Received 15 November 2017; revised manuscript received 17 January 2018; published 2 April 2018)

\begin{abstract}
The noncommutativity of position and momentum observables is a hallmark feature of quantum physics. However, this incompatibility does not extend to observables that are periodic in these base variables. Such modular-variable observables have been suggested as tools for fault-tolerant quantum computing and enhanced quantum sensing. Here, we implement sequential measurements of modular variables in the oscillatory motion of a single trapped ion, using state-dependent displacements and a heralded nondestructive readout. We investigate the commutative nature of modular variable observables by demonstrating no-signaling in time between successive measurements, using a variety of input states. Employing a different periodicity, we observe signaling in time. This also requires wave-packet overlap, resulting in quantum interference that we enhance using squeezed input states. The sequential measurements allow us to extract two-time correlators for modular variables, which we use to violate a Leggett-Garg inequality. Signaling in time and Leggett-Garg inequalities serve as efficient quantum witnesses, which we probe here with a mechanical oscillator, a system that has a natural crossover from the quantum to the classical regime.
\end{abstract}

DOI: 10.1103/PhysRevX.8.021001

Subject Areas: Quantum Physics

\section{INTRODUCTION}

One of the fundamental notions of quantum mechanics is that position and momentum operators do not commute. This results in the Heisenberg uncertainty principle: $\Delta \hat{x} \Delta \hat{p} \geq \frac{1}{2}|\langle[\hat{x}, \hat{p}]\rangle|$ with $[\hat{x}, \hat{p}]=i \hbar$, restricts the possible states of a particle and limits the ability to perform simultaneous position and momentum measurements [1-4]. However, this is different for measurements of the position and momentum operator modulo a characteristic length or momentum scale (i.e., $\hat{X} \bmod l_{x}, \hat{P} \bmod l_{p}$ ), which can commute [5]. Such operators were first discussed in the context of the seminal Aharonov-Bohm effect [6], and they provide new perspectives in the study of fundamental aspects of quantum mechanics. For example, they exhibit nonlocal Heisenberg equations of motion [7]. Modular operators have been proposed for testing macrorealism via Leggett-Garg inequalities (LGI) [8] as well as contextuality with continuous-variable systems [9]. The

\footnotetext{
* Corresponding author. christaf@phys.ethz.ch

Corresponding author. jhome@phys.ethz.ch

Published by the American Physical Society under the terms of the Creative Commons Attribution 4.0 International license. Further distribution of this work must maintain attribution to the author(s) and the published article's title, journal citation, and DOI.
}

commutation of modular position and momentum operators allows their use as stabilizers for fault-tolerant continuous-variable computation, as proposed by Gottesman, Kitaev, and Preskill (GKP) [10]. The GKP encoding has recently been shown to outperform alternative bosonic codes [11]. Additionally, sequences of measurements of these modular operators have been proposed to prepare approximate GKP code states [12,13]. In contrast, for incompatible modular position and momentum measurement settings, we expect the first measurement to influence the statistics of the subsequent measurement, which has previously been defined as signaling in time (SIT) [14,15]. Observation of signaling in time and Leggett-Garg inequality violations provide means to exclude macrorealistic theories and often serve as quantum witnesses [14-17].

In this paper, we implement and analyze sequences of modular position and momentum measurements of a quantum harmonic oscillator realized in the axial motional oscillation at $\omega \approx 2 \pi \times 1.85 \mathrm{MHz}$ of a single trapped ${ }^{40} \mathrm{Ca}^{+}$ atomic ion. The observables are measured by coupling the oscillator to the ion's internal qubit states using statedependent forces and subsequently reading out the qubit using resonance fluorescence [18]. We analyze signaling in time between the measurements and violate a Leggett-Garg inequality. Using both methods, we confirm the quantum nature of the motional states using a small number of measurements. Furthermore, the sequential measurements allow us to test the commutation of modular position 
and momentum operators, which we do by observing nosignaling in time (NSIT) on a variety of input states. The experiments demonstrate control and show coherence of multicomponent superpositions of up to eight coherent, squeezed, or Fock state wave packets.

\section{GENERAL OSCILLATOR MEASUREMENT}

A measurement of a physical system involves coupling to a second system - the meter-which is interpreted as providing classical output [19]. To precisely define the consequences of the coupling, quantum systems should be used for both the system and the meter. In the presented experiments, the system is coupled via a unitary transformation $\hat{V}$ to a secondary quantum system (a qubit), which is subsequently read out via a projective binary measurement in the $\hat{\sigma}_{z}$ basis given by the $|\downarrow\rangle,|\uparrow\rangle$ states. Such a measurement is described by the quantum circuit in Fig. 1(a). The important questions for a measurement are as follows: (i) What are the probabilities for the different outcomes? (ii) How is the system modified? (iii) What do the outcomes reveal about the system?

To answer these questions, we consider the unitary transformation $\hat{V}$ given in Fig. 1(a) applied to an input system state $\left|\psi_{\text {in }}\right\rangle$ with the meter qubit initialized to $|\downarrow\rangle$. This results in an entangled state between system and meter given by $|\uparrow\rangle \otimes \hat{E}_{-}\left|\psi_{\text {in }}\right\rangle+|\downarrow\rangle \otimes \hat{E}_{+}\left|\psi_{\text {in }}\right\rangle$, with $\hat{E}_{ \pm}=\hat{U}(\hat{S} \pm \mathbb{1}) / 2$. The outcomes of the subsequent projective qubit measurement are \pm 1 , corresponding to the eigenstates of $\hat{\sigma}_{z}$. The corresponding measurement (Kraus) operators are $\hat{E}_{ \pm}-$ these completely define all properties of the measurement. The probabilities to obtain each measurement outcome are given by $P( \pm 1)=\left\langle\psi_{\text {in }}\left|\hat{E}_{ \pm}^{\dagger} \hat{E}_{ \pm}\right| \psi_{\text {in }}\right\rangle$, while the quantum state after the measurement is $\left|\psi_{ \pm}\right\rangle=\hat{E}_{ \pm}\left|\psi_{\text {in }}\right\rangle / \sqrt{P( \pm 1)}$. The expectation value of the qubit operator $\left\langle\hat{\sigma}_{z}\right\rangle=$ $(+1) P(+1)+(-1) P(-1)=\left\langle\psi_{\text {in }}\left|\left(\hat{E}_{+}^{\dagger} \hat{E}_{+}-\hat{E}_{-}^{\dagger} \hat{E}_{-}\right)\right| \psi_{\text {in }}\right\rangle=$ $\left\langle\psi_{\text {in }}|\hat{Q}| \psi_{\text {in }}\right\rangle$, where $\hat{Q} \equiv \hat{E}_{+}^{\dagger} \hat{E}_{+}-\hat{E}_{-}^{\dagger} \hat{E}_{-}$. By the act of measurement, we obtain (partial) information about the observable represented by the operator $\hat{Q}$ [19]. The extracted information does not depend on the unitary transformation $\hat{U}$, although this does affect the state of the system after the measurement.

The type of measurement circuit shown in Fig. 1(a) is prominently featured in quantum error correction, where it is often used to read out stabilizer operators [20]. The stabilizer operator $\hat{S}$ is Hermitian and has two discrete eigenvalues \pm 1 ; thus, in the case of $\hat{U}=\mathbb{1}$, the measurement operators $\hat{E}_{ \pm}$are projectors onto the corresponding eigenspaces, while the readout observable $\hat{Q}=\hat{S}$. In contrast, the experiments considered in this paper use a harmonic oscillator as the system of interest with a coupling given by $\hat{S}=e^{i \phi} \hat{\mathcal{D}}(\alpha)$, where $\hat{\mathcal{D}}(\alpha)$ is an oscillator phase-

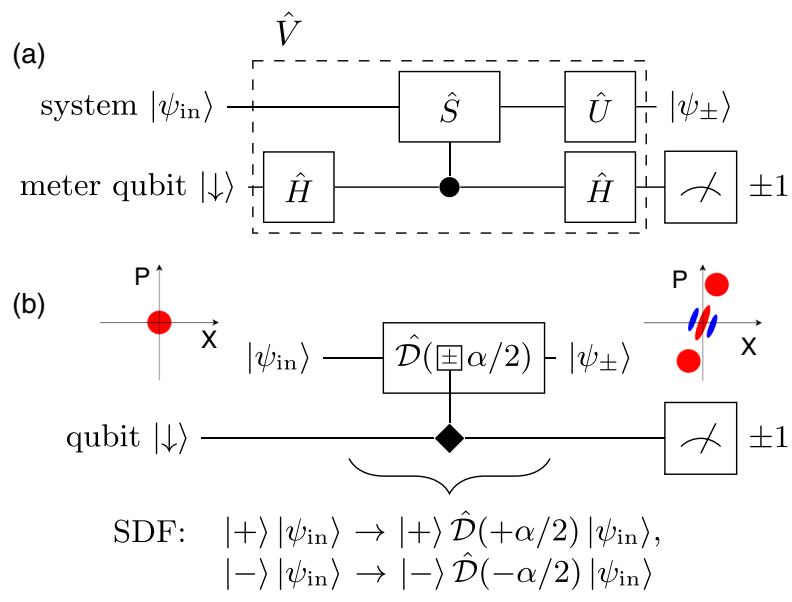

FIG. 1. (a) Circuit model of the measurement type used in this paper. In general, a measurement proceeds by coupling the system of interest to a meter via a unitary transformation $\hat{V}$ (enclosed in the dashed box). Our measurements use a qubit as the meter system, and a common coupling is given by a controlled-unitary $(\hat{S})$. This gives a phase kickback on the meter qubit depending on the eigenvalue of the operator $\hat{S}$. The phase change is converted to a qubit population by applying the controlled operation between two Hadamard gates $(\hat{H})$. The action of $\hat{V}$ may involve additional unitary transformations $\hat{U}$, which affect the post-measurement state but not the measurement outcome. (b) In our experiments, the system of interest is a harmonic oscillator. The core element is a spin-state-dependent force (SDF), which realizes a phase-space displacement $\hat{\mathcal{D}}( \pm \alpha / 2)$, with the displacement sign dependent on the $\hat{\sigma}_{x}$ eigenstate of the qubit. This is denoted with the diamond control. This realizes a circuit of the type shown in diagram (a) with $\hat{S}=-\hat{\mathcal{D}}(\alpha)$ and $\hat{U}=\hat{\mathcal{D}}(-\alpha / 2)$. Shown as input is the Wigner function of the oscillator ground state, which is projected into a "Schrödinger's cat" like superposition by the measurement with the relative phase of the two components correlated with the measurement outcome.

space displacement by the complex parameter $\alpha$ [21]. Since the displacement operator has a continuous eigenvalue spectrum, a single binary measurement cannot extract its eigenvalue, and the $\hat{E}_{ \pm}$are no longer projection operators. The measurement nevertheless gives partial information about the related operator $\hat{Q}$, which is given by

$$
\hat{Q}(\phi, \alpha)=\cos (\phi+2 \operatorname{Im}(\alpha) \hat{X}-2 \operatorname{Re}(\alpha) \hat{P}),
$$

where we define $\hat{X}=\sqrt{m \omega /(2 \hbar)} \hat{x}$ and $\hat{P}=\sqrt{1 /(2 m \omega \hbar)} \hat{p}$ as dimensionless position and momentum operators, with $\omega$ and $m$ denoting the harmonic oscillator frequency and mass. For this definition, $[\hat{X}, \hat{P}]=i / 2$. Here, $\hat{Q}$ is a modular function of the position and momentum operators, and it is closely related to $\hat{X} \bmod l_{x}, \hat{P} \bmod l_{p}$ [22]. To obtain the eigenvalue of $\hat{\mathcal{D}}(\alpha)$ up to a given precision, phase 
estimation protocols using multiple measurement rounds would be required. In such protocols, the initial system state in each round is the post-measurement state of the previous round, and the backaction of each measurement pushes the system towards an eigenstate of $\hat{\mathcal{D}}(\alpha)$ [4].

The unitary transformation $\hat{U}$ is related to the experimental implementation of the measurement. In our apparatus, we can implement the circuit with $\hat{U}=\hat{\mathcal{D}}(-\alpha / 2)$ and $\phi=\pi$, with a single laser pulse as described in Sec. III. We call this the "symmetric" implementation, and we use it for the measurements described in Secs. IV and V. Additional flexibility can be added by using a sequence of pulses involving an additional internal state level of our ion (Appendix C). This implements the circuit with $\hat{U}=\mathbb{1}$, which we call the "asymmetric" implementation and which is used for the experiments in Secs. VI and VII.

\section{IMPLEMENTATION OF SEQUENTIAL MODULAR MEASUREMENTS}

The symmetric modular measurements are implemented using a bichromatic laser field resonant with both the red and blue sidebands of the quadrupole transition between the $|\downarrow\rangle \equiv\left|S_{1 / 2}, m_{j}=1 / 2\right\rangle$ and $|\uparrow\rangle \equiv\left|D_{5 / 2}, m_{j}=3 / 2\right\rangle$ internal states [23]. This realizes a Hamiltonian $\hat{H}_{\mathrm{SDF}}=\eta \hbar \Omega \hat{\sigma}_{x}\left(\hat{a} e^{i \Delta \phi / 2}+\hat{a}^{\dagger} e^{-i \Delta \phi / 2}\right) / 2$, where $\hat{\sigma}_{x} \equiv$ $|\uparrow\rangle\langle\downarrow|+| \downarrow\rangle\langle\uparrow|, \eta \simeq 0.05$ is the Lamb-Dicke parameter [18], $\hat{a}$ is the harmonic oscillator destruction operator, and $\Omega, \Delta \phi$ are related to the intensity and relative phases of the sideband laser fields. The corresponding time evolution operator is $\hat{\mathcal{D}}\left(\alpha(t) \hat{\sigma}_{x} / 2\right)$, where $\alpha(t)=i e^{i \Delta \phi / 2} \eta \Omega t$. This state-dependent force (SDF) thus displaces the oscillator in opposite directions dependent on the internal qubit state being in one or the other of the Pauli- $\hat{\sigma}_{x}$ operator eigenstates [see Fig. 1(b)].

In the ideal scenario, the measurement projects into the state $\left|\psi_{ \pm}\right\rangle \propto \hat{E}_{ \pm}\left|\psi_{\text {in }}\right\rangle$, conditional on the measurement result, which is a superposition of two displaced copies of $\left|\psi_{\text {in }}\right\rangle$. For example, an initially ground-state cooled oscillator is projected into a Schrödinger's cat-type superposition, with a relative phase dependent on the measurement result [see Fig. 1(b)]. In practice, we measure the qubit using state-dependent resonance fluorescence, which for the detection of $|\uparrow\rangle$ (no photons scattered), closely realizes the ideal scenario. However, measuring the $|\downarrow\rangle$ state involves scattering of around 1000 photons, which randomizes the oscillator state. We thus perform the measurement in a heralded fashion and only analyze the subsequent state (or continue to further measurements) if the detection is dark. This decision is made in real time using a field programmable gate array to save dataacquisition time. In half of our experiments, we invert the qubit prior to the fluorescence detection, allowing projection into $\left|\psi_{+}\right\rangle \propto \hat{E}_{+}\left|\psi_{\text {in }}\right\rangle$ as a dark measurement result.

\section{SIGNALING IN TIME}

In quantum mechanics, the measurement of one quantity often influences a subsequent measurement of a different quantity. We analyze such effects using two symmetric modular variable measurements $A, B$, with measurement settings controlled through the respective displacements $\alpha_{A}$ and $\alpha_{B}$. The measurement outcomes are $a, b \in\{+1,-1\}$. Measurement $B$ is either performed alone or subsequent to a measurement of $A$, resulting in probabilities $P_{B}(b)$ or $P_{B(A)}(b) \equiv \sum_{a} P_{B A}(b, a)$, respectively, where we have defined the joint probability $P_{B A}(b, a) \equiv P_{A}(a) P_{B \mid A}(b \mid a)$. For some settings, the statistics of measurement $B$ change if measurement $A$ is performed before it. We call this signaling in time (SIT). Since the measurement is binary, we can quantify SIT of $A$ to $B$ using $S=P_{B}(b=+1)-$ $P_{B(A)}(b=+1)$, which, for our experiments, results in (Appendix G)

$$
S=\frac{1}{2}(1-\cos (\Phi))\left|m_{\alpha_{B}}\right| \cos \left(\arg \left(m_{\alpha_{B}}\right)\right),
$$

where $m_{\alpha} \equiv\left\langle\psi_{\text {in }}|\hat{\mathcal{D}}(\alpha)| \psi_{\text {in }}\right\rangle$ and $\Phi=\operatorname{Im}\left(\alpha_{A}^{*} \alpha_{B}\right)$ is the geometric phase that arises from the noncommutation of the displacement operators $\hat{\mathcal{D}}\left(\alpha_{A}\right) \hat{\mathcal{D}}\left(\alpha_{B}\right)=e^{i \Phi} \hat{\mathcal{D}}\left(\alpha_{A}+\alpha_{B}\right)$ [21]. If for some settings the statistics of measurement $B$ do not depend on the presence of measurement $A$, i.e., $S=0$, then we say measurement $A$ is NSIT to measurement $B$. From this expression, we see that SIT will not occur for any state if either the geometric phase $\Phi=2 \pi k, k \in \mathbb{Z}$ or the wave-packet overlap $\left|m_{\alpha_{B}}\right|=0$. We analyze these dependencies in two experiments.

We examine the effect of wave-packet overlap using squeezed vacuum states $\left|\psi_{\text {in }}\right\rangle=\hat{S}(r)|0\rangle$ with $\hat{S}(r)=$ $e^{r\left(\hat{a}^{2}-\hat{a}^{\dagger 2}\right) / 2}$ and where the phase is chosen such that the squeezing parameter $r$ is real and positive. By changing the squeezing parameter $r$, we can control the extent of the oscillator state in phase space, which in turn affects the wave-packet overlap. The squeezed states can be readily prepared using reservoir engineering [24]. We choose the measurement displacement $\alpha_{B}=3.1 i$, which is thus aligned with the antisqueezed axis of the squeezed input state. The wave-packet interference in this case scales as $m_{\alpha_{B}}=e^{-\left|\alpha_{B}\right|^{2} e^{-2 r} / 2}$ [25]. We choose $\alpha_{A}=3.02 \approx 3 \pi /\left|\alpha_{B}\right|$ to ensure that, for a given overlap, maximal SIT is observed. Experimental results are shown in Fig. 2(a), exhibiting agreement with the ideal theoretical expectation. Deviations between the two for large $r$ are primarily due to imperfect squeezed state preparation.

The input state $\left|\psi_{\text {in }}\right\rangle \propto\left(\hat{\mathcal{D}}\left(-\alpha_{B} / 2\right)+\hat{\mathcal{D}}\left(\alpha_{B} / 2\right)\right)|0\rangle$ exhibits a constant nonzero level of interference $\left|m_{\alpha_{B}}\right| \approx 1 / 2$. Thus, we use this state to illustrate the dependence of SIT on the geometric phase. This is done by varying $\alpha_{A}$, which is taken to be real. The measured data points are displayed 

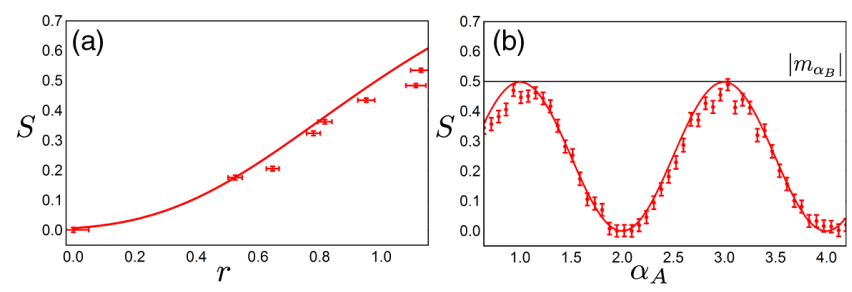

FIG. 2. Dependence of SIT of measurement $A$ to measurement $B$ on (a) interference and (b) geometrical phases. Solid lines show the expectations for an ideal experimental system, and the error bars of $S$ are propagated from the shot-noise standard errors of the mean (SEM) of the directly measured probabilities $P_{B}(b), P_{A}(a)$, and $P_{B \mid A}(b \mid a)$. (a) The SIT measurement settings $\alpha_{B}=3.1 i$, $\alpha_{A}=3.02 \approx 3 \pi /\left|\alpha_{B}\right|$ are applied to squeezed input states $\hat{S}(r)|0\rangle$, where the squeezed axis is aligned with position. (b) The geometric phase is varied by sweeping the displacement amplitude $\alpha_{A}$ of measurement $A$, and using a Schrödinger's cat input superposition $\left(\hat{\mathcal{D}}\left(-\alpha_{B} / 2\right)+\hat{\mathcal{D}}\left(\alpha_{B} / 2\right)\right)|0\rangle$ with $\alpha_{B}=i \pi$.

in Fig. 2(b), where we see that the measured amount of SIT oscillates as a function of $\alpha_{A}$ with an amplitude $\left|m_{\alpha_{B}}\right|$. These oscillations illustrate the periodic effect of the geometric phase. NSIT is seen for this measurement when $\alpha_{A}=2 \pi k /\left|\alpha_{B}\right| \approx 2 k$.

\section{COMMUTATION OF MODULAR MOMENTUM AND POSITION MEASUREMENTS}

If NSIT is observed for all possible input states, then it follows that the underlying observables commute. The converse is not true for the nonprojective measurements considered here (see Appendix I). The commutation of observables is hard to verify in practice, given the infinite nature of the harmonic oscillator Hilbert space. As a reduced investigation, we examine this property using 150 input states of the form $\left|\psi_{\text {in }}\right\rangle \propto\left(\hat{\mathcal{D}}\left(-\left|\alpha_{B}\right| e^{i \phi_{I}} / 2\right)+\right.$ $\left.\hat{\mathcal{D}}\left(\left|\alpha_{B}\right| e^{i \phi_{I}} / 2\right)\right)|\phi\rangle$, where $|\phi\rangle$ is chosen to be one of (i) the ground state $|0\rangle$, (ii) a squeezed state $\hat{S}(-0.82)|0\rangle$, or (iii) the first excited state $|1\rangle$; for each $|\phi\rangle, 50$ values of $\phi_{I}$ evenly spaced between zero and $2 \pi$ are used. To investigate the commutation of modular position and momentum for large displacements, we choose the NSIT geometric phase with $k=2(\Phi \approx 4 \pi)$, which we implement using the measurement settings: $\alpha_{B}=i \pi, \alpha_{A}=4.09$. For these settings, the modular measurement observables are also expected to commute (see Appendix I).

Data and a histogram of all measured values of $S$ are shown in Figs. 3(a)-3(c). For comparison, in (c) we display the theoretical histogram for $\alpha_{B}=i \pi, \alpha_{A}=3$, resulting in $\Phi=3 \pi$, which corresponds to maximal SIT but with the same $m_{\alpha_{B}}$ as used in the experiment. The theoretical calculation was performed for the same 150 input states as were used in the experiment. The maximal $|S|$ value measured is $0.087 \pm 0.003$, while the maximum value calculated is 0.5 . Additionally, the standard deviation of (a) Measurement $B$
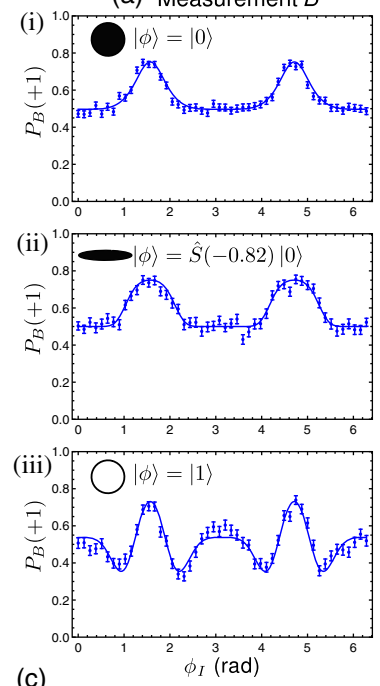

(c)
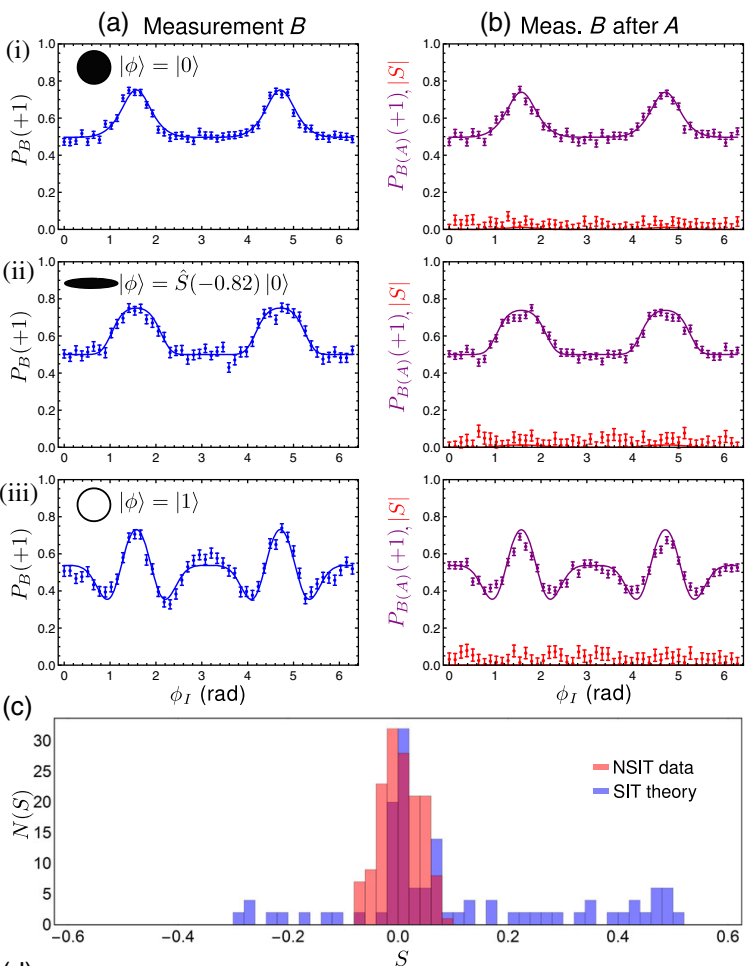

(d)
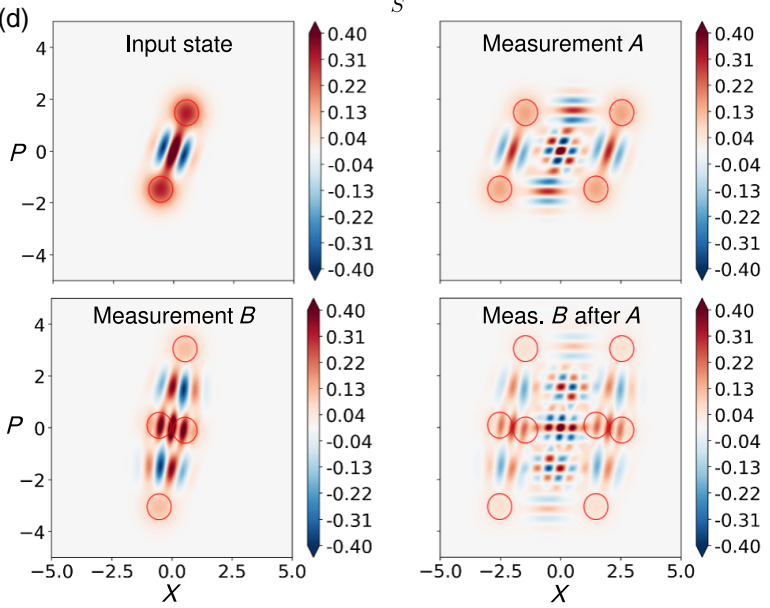

FIG. 3. NSIT of modular position and momentum measurements for a variety of input states. Rows (i)-(iii) show NSIT of $A$ to $B$ for the measurement settings $\alpha_{B}=i \pi, \alpha_{A}=4.09 \approx 4 \pi /\left|\alpha_{B}\right|$, and input superposition states $\left(\hat{\mathcal{D}}\left(-\left|\alpha_{B}\right| e^{i \phi_{I}} / 2\right)+\hat{\mathcal{D}}\left(\left|\alpha_{B}\right| e^{i \phi_{I}} / 2\right)\right)|\phi\rangle$ with the phase $\phi_{I}$ varied. Here, $|\phi\rangle$ is chosen to be (i) the ground state $|0\rangle$, (ii) a squeezed vacuum state $\hat{S}(-0.82)|0\rangle$, or (iii) the first excited state $|1\rangle$. We observe qualitative agreement between column (a), showing measurement of $B$ alone, and column (b), showing $B$ measured after $A$. Solid lines show the expectations for an ideal experiment, which are identical in both columns. Errors are given as SEM and propagation of SEM. The 150 measured $S$ values are quantified in red in histogram (c) and compared to a theoretical histogram for the SIT settings $\alpha_{A}=3$ and $\alpha_{B}=i \pi$ using the same set of input states (see main text). (d) Theoretical Wigner function plots of the input state $(|\phi\rangle=|0\rangle$ and $\phi_{I}=1.22 \mathrm{rad}$ ) as well as its post-measurement states with result +1 during the experimental sequence. The red circles show the locations of the multiple displaced coherent states; their radius denotes the r.m.s. wave-packet size. 
the SIT theory histogram is 5.5 times larger than that of the experimentally measured distribution. Theoretical Wigner function plots for one input state example $(|\phi\rangle=|0\rangle$ and $\left.\phi_{I}=1.22 \mathrm{rad}\right)$, throughout the experimental sequence, are shown in Fig. 3(d). The created states are superpositions of up to eight displaced $|\phi\rangle$ states, with separations of up to $\Delta \alpha \approx 8.3$. These measurements illustrate the high level of control for the implemented sequential modular measurements. The ability to tune from SIT to NSIT demonstrates the quantum nature of the created states and additionally confirms the possibility of modular position and momentum measurements to commute.

\section{TWO-TIME CORRELATORS}

An additional means by which successive measurements can be related to one another is through the correlation function of the measurement results, which is defined by $C_{A B}=\sum_{a, b} a b P_{B A}(b, a)$. For the measurements described above, the correlation function between the two measurements is $C_{A B}=\left(\left|m_{\alpha_{A}-\alpha_{B}}\right| \cos \left(\varphi_{-}\right)+\left|m_{\alpha_{A}+\alpha_{B}}\right| \cos \left(\varphi_{+}\right)\right) / 2$, with $\varphi_{ \pm}=\arg \left(m_{\alpha_{A} \pm \alpha_{B}}\right)$. This is independent of the geometric phase $\Phi$, which was required to be nonzero in order to observe SIT.

To investigate the effect of the geometric phase on correlations, in the following, we explore the asymmetric modular measurement implementation with measurement operators $\hat{F}_{ \pm}(\phi, \alpha)=\frac{1}{2}\left(\mathbb{1} \pm e^{i \phi} \hat{\mathcal{D}}(\alpha)\right)$. For this implementation, we expect $\tilde{S}=\sin (\Phi)\left|m_{\alpha_{B}}\right| \sin \left(\Phi+\phi_{B}+\arg \left(m_{\alpha_{B}}\right)\right)$, and the correlation function is (derivation in Appendix G)

$$
\begin{aligned}
\tilde{C}_{A B} & =\frac{1}{2}\left(\left|m_{\alpha_{A}-\alpha_{B}}\right| \cos \left(\tilde{\varphi}_{-}\right)+\left|m_{\alpha_{A}+\alpha_{B}}\right| \cos \left(\tilde{\varphi}_{+}\right)\right) \\
\tilde{\varphi}_{ \pm} & =\phi_{A} \pm \phi_{B} \pm \Phi+\arg \left(m_{\alpha_{A} \pm \alpha_{B}}\right) .
\end{aligned}
$$

This implementation thus reintroduces the geometric phase to the correlator. It is experimentally achieved using a third energy level in the ion (see Appendix C). The observables of the two implementations are the same, but the postmeasurement states differ. This changes the result of the second measurement and thus the time correlator and the signaling-in-time parameter $\hat{S}$.

A measurement of the correlation function using the asymmetric implementation as a function of $\alpha_{B}$ is shown in Fig. 4(a). The experimental parameters were $\alpha_{A}=2.1$, $\phi_{A}=0, \phi_{B}=\pi / 2$, and the input state was the ground state $\left|\psi_{\text {in }}\right\rangle=|0\rangle$. The correlation function then reads $\tilde{C}_{A B}=-\left(e^{-\left|2.1-\alpha_{B}\right|^{2} / 2}+e^{-\left|2.1+\alpha_{B}\right|^{2} / 2}\right) \sin (\Phi) / 2$, with the geometric phase $\Phi=2.1 \operatorname{Im}\left(\alpha_{B}\right)$. The prefactor is nonzero for $\alpha_{B} \approx \pm 2.1$. In this case, wave packets overlap in the post-measurement state of $B$, leading to interference effects during the measurement. The sign change of the correlator in this example is solely due to the geometric phase $\Phi$. The extreme values of $\tilde{C}_{A B}$ are reached as a compromise between the wave-packet overlap and the geometric phase.

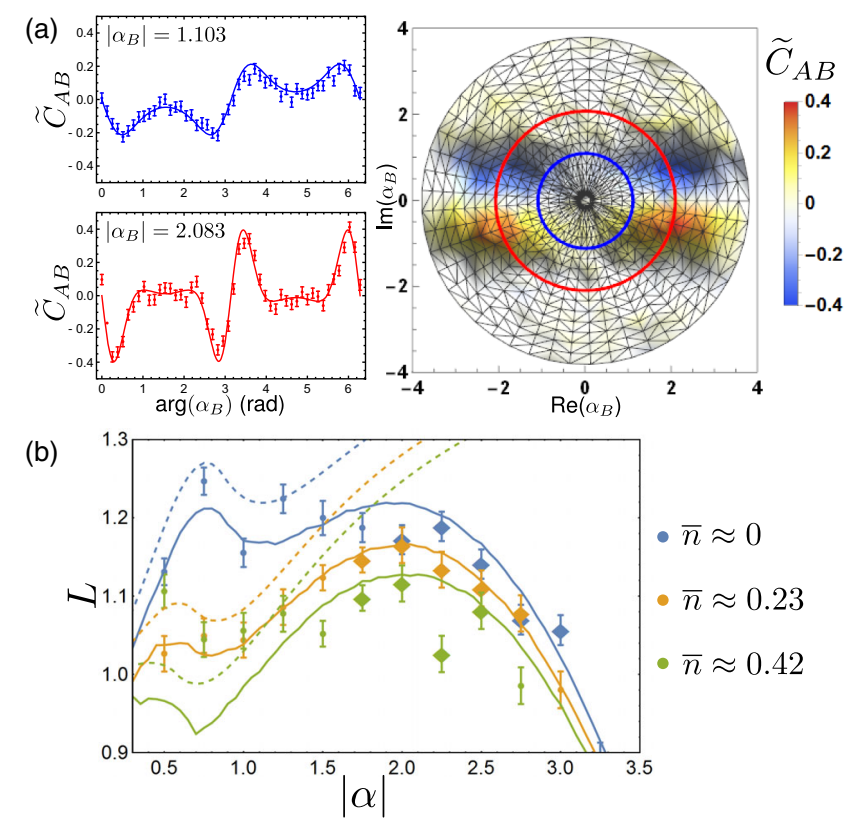

FIG. 4. Two time correlators and violation of a Leggett-Garg inequality. (a) Two-time correlation measurement in the asymmetric implementation performed on an initial ground-state cooled ion as a function of the second displacement $\alpha_{B}$. The fixed experimental settings were $\alpha_{A}=2.1, \phi_{A}=0, \phi_{B}=\pi / 2$. The full data set is shown in the false-color plot to the right. Two cuts through this data set with fixed $\left|\alpha_{B}\right|$ are indicated in this false-color plot and explicitly plotted to the left, where solid lines show the expectations for an ideal experiment. (b) Violation of a Leggett-Garg inequality for increased modular measurement displacements $|\alpha|$ and three different initial temperatures. Solid lines show the expected violation including simulated qubit and motional dephasing as well as phase calibration errors (see Appendix N); the dashed lines instead are the exceptions for an ideal experiment. Violations are observed over a wide range of $\alpha$ for all investigated temperatures. With a ground-state cooled ion, violations are observed up to $\alpha=3$. The points highlighted with a diamond violate the LGI when being penalized by the built-in theoretical amount of SIT (Appendix O). The discrepancy between the data and the simulation at $\bar{n}=0.42$ is due to additional experimental fluctuations in the preparation of this higher thermal occupation. All error bars are propagated from the SEM errors because of quantum projection noise.

\section{VIOLATION OF LEGGETT-GARG INEQUALITY WITH A MECHANICAL HARMONIC OSCILLATOR}

Correlation functions lie at the heart of many tests of the quantum nature of physical systems [17,26,27]. For systems measured at sequential times, the best-known test is the violation of the LGI, for which one form is given by

$$
L=C_{A B}+C_{B C}-C_{A C} \leq 1
$$

where a time sequence of three measurements $A, B, C$ is considered. The bound is derived under two assumptions: 
(i) These measurements are of macroscopic quantities with preexisting values, and (ii) these values are unchanged by the act of measurement [17]. Therefore, to exclude macroscopic realism, noninvasive measurements need to be used in the experiment, which is hard to ensure in practice.

A protocol for testing LGI using modular variable measurements has been proposed previously [8], where it was shown that violation of the LGI can be used to differentiate between an oscillator described by a classical variable and a quantum mechanical oscillator. This is a weaker statement than excluding macrorealism, and it allows us to drop the original requirement of noninvasive measurements. The classical variable does not allow the observation of SIT. Thus, the observation of SIT or LGI violations can be used to confirm the presence of states showing quantum features. In previous work, revivals and oscillations of qubit excitation in single-time detections performed over a range of settings were taken as an indirect measure for the creation of quantum superposition states $[25,28,29]$. These single-time detection features could, however, be produced by the coupling to an adequate classical field distribution (see example in Appendix L), which is not the case for LGI violations or measurements of SIT based on sequential measurements.

To measure $L$ for our oscillator, we measure two-time correlations between each pair of three modular measurements $A, B, C$ while leaving out the third [8]. The modular displacement settings used for the measurements can be parametrized as $\alpha_{A}=|\alpha| e^{i \theta_{A}}, \alpha_{B}=|\alpha| e^{i \theta_{B}}, \alpha_{C}=|\alpha| e^{i \theta_{C}}$, with the respective angles $\theta_{A}=\omega t_{1}, \theta_{B}=\omega t_{2}, \theta_{C}=\omega t_{3}$ arranged to meet the constraints of successive measurements at times $t_{1}, t_{2}, t_{3}$. For a fixed $\alpha$ and an initial thermal state of the oscillator, we numerically find values for $\theta_{A}, \theta_{B}$, and $\theta_{C}$ and the phases $\phi_{A}, \phi_{B}$, and $\phi_{C}$, which maximize the expected value of $L$, and use these for the experiments. Here, $L$ was measured for three thermal input states with average occupations $\bar{n} \approx 0, \bar{n} \approx 0.23$, and $\bar{n} \approx 0.42$. This allows us to test the robustness of the protocol with respect to finite thermal occupations. Results are given in Fig. 4(b), showing $L>1$ for displacements up to $\alpha=3$. We notice that $L$ is sensitive to noise in the experimental implementation because it involves measuring three extremal correlations. The dashed lines in Fig. 4(b) show the expected violations for an ideal experiment, and the solid lines show simulations using the level of motional and qubit dephasing that was observed in previous experiments performed in the same apparatus [30]. Spin decoherence limits the violation at small $\alpha$, and the sharp drop in violation above $\alpha=2$ is caused by motional dephasing.

\section{RELATION BETWEEN SIGNALING IN TIME AND LEGGETT-GARG INEQUALITY QUANTUM WITNESSES}

For any experiment in which SIT is observed, protocols can be designed for which the Leggett-Garg inequality is violated (see Appendix Q). NSIT was previously discussed as a means to experimentally test the noninvasive measurement condition needed to exclude macrorealism [14], and efforts have been undertaken to improve the LGI test by adding additional NSIT constraints and exploring their implications [31]. An alternative route is to penalize the value of $L$ by accounting for SIT between the measurements [32]. The protocol we implement approaches NSIT for large displacements. If we penalize our measurement values using the theoretical value of SIT expected for our settings, only the data points highlighted by diamonds in Fig. 4(b) produce a LGI violation (see Appendix O for further details). For fixed displacement, the performance of the LGI protocol could be improved in the future using squeezed input oscillator states. This leads to experimentally more robust measurements, larger ratios of separation to r.m.s. wave-packet size, and thus less SIT between the measurements (see Appendix S).

\section{CONCLUSION}

The measurement techniques demonstrated here provide new tools for examining the quantum-classical divide with harmonic oscillators and could be applied in a range of experimental systems [8]. Both the Leggett-Garg correlation method and signaling in time provide quantum signatures using few measurements, although we find experimentally that they require excellent frequency stability of the oscillator mode. Extensions to multiple oscillators would allow tests of local realism and noncontextuality with continuous variables [33]. Alongside these fundamental applications, the combination of squeezed states and modular operator measurements demonstrated here could be used to prepare approximate oscillator-error-correction code states as proposed by Gottesman, Kitaev, and Preskill [10,12,13]. Ideal code states would exhibit maximal signaling in time. The control demonstrated here provides a toolbox for investigating these fault-tolerant schemes, opening up a new path to large-scale quantum computing with continuous variables.

\section{ACKNOWLEDGMENTS}

We thank Thanh Long Nguyen for carefully checking the manuscript, and Peter Rabl, Renato Renner, Joseba Alonso, and Maciej Malinowski for useful discussions. We acknowledge support from the Swiss National Science Foundation under Grant No. 200021_134776, ETH Research Grant No. ETH-18 12-2, and from the Swiss National Science Foundation through the National Centre of Competence in Research for Quantum Science and Technology (QSIT). The research is partly based upon work supported by the Office of the Director of National Intelligence (ODNI), Intelligence Advanced Research Projects Activity (IARPA), via the U.S. Army Research Office Grant No. W911NF-16-1-0070. The views and 
conclusions contained herein are those of the authors and should not be interpreted as necessarily representing the official policies or endorsements, either expressed or implied, of the ODNI, IARPA, or the U.S. Government. The U.S. Government is authorized to reproduce and distribute reprints for Governmental purposes notwithstanding any copyright annotation thereon. Any opinions, findings, and conclusions or recommendations expressed in this material are those of the author(s) and do not necessarily reflect the view of the U.S. Army Research Office.

Experimental data were taken and analyzed by C.F., using an apparatus with significant contributions from V. N., M. M., and C.F. The paper was written by C.F. and J.P. H., with input from all authors. Experiments were conceived by C. F. and J. P. H.

The authors declare that they have no competing financial interests.

\section{APPENDIX A: DEFINITION OF DIMENSIONLESS POSITION AND MOMENTUM}

We choose definitions of dimensionless position and momentum such that we have a simple connection to phase space: $\hat{X}=\sqrt{[(m \omega) /(2 \hbar)]} \hat{x}$ and $\hat{P}=\sqrt{[1 /(2 m \omega \hbar)]} \hat{p}$ lead to $\langle\alpha|\hat{X}| \alpha\rangle=\operatorname{Re}(\alpha),\langle\alpha|\hat{P}| \alpha\rangle=\operatorname{Im}(\alpha)$, and $[\hat{X}, \hat{P}]=i / 2$. This definition simplifies working with position, momentum, and displacement operators simultaneously.

\section{APPENDIX B: QUBIT READOUT}

In a temporal sequence of measurements, the last measurement is performed as a long fluorescence detection with a typical readout time of $200 \mu$ s. All the preceding measurements are performed with a shorter fluorescence time of $60 \mu \mathrm{s}$, having a detection error of $\epsilon_{\text {short }} \approx 4 \times 10^{-3}$ and giving an average of roughly 10 counts for a bright detection result. Our imaging system collects $4.4 \%$ of the emitted photons, and the PMT quantum efficiency is approximately $26.5 \%$. This amounts to an average scattering of 1000 photons from the ion for this shorter detection.

\section{APPENDIX C: PULSE SEQUENCE REALIZING THE ASYMMETRIC IMPLEMENTATION}

We implement the asymmetric modular measurements making use of three internal energy levels. Besides the levels $|\downarrow\rangle,|\uparrow\rangle$, we additionally use a second level in the $D_{5 / 2}$ manifold $|a\rangle \equiv\left|D_{5 / 2}, m_{j}=-1 / 2\right\rangle$. The measurement is implemented with the sequence of operations (read right to left) $\hat{R}_{1}(\phi) \hat{R}_{2}(0) \hat{\mathcal{D}}\left(\alpha(t) \hat{\sigma}_{x, 2}\right) \hat{R}_{2}(\pi) \hat{R}_{1}(0)$, using the definitions $\hat{\mathcal{D}}(\alpha)=e^{\alpha \hat{a}^{\dagger}-\alpha^{*} \hat{a}}$ and $\hat{R}_{k}(\varphi)=$ $1 / \sqrt{2}\left(\mathbb{1}-i \sin (\varphi) \hat{\sigma}_{k, x}+i \cos (\varphi) \hat{\sigma}_{k, y}\right)$. The Pauli matrices $\hat{\sigma}_{k}$ are taken to act on the $|a\rangle,|\downarrow\rangle$ basis for $k=1$ and $|\downarrow\rangle,|\uparrow\rangle$ basis for $k=2$. Spin rotations are implemented using resonant pulses on the two transitions, while the statedependent displacement uses a bichromatic laser field resonant with both the red and blue sidebands of transition $k=2[23]$.

If the pulse sequence is applied to an ion initially in the $|\downarrow\rangle$ level, then the first pulse puts half the population in the $|a\rangle$ state. This part of the population is then not affected by the block of operations $\hat{R}_{2}(0) \hat{\mathcal{D}}\left(\alpha(t) \hat{\sigma}_{x, 2}\right) \hat{R}_{2}(\pi)$, which acts on transition 2. In this block, the two rotations around the SDF pulse effectively rotate the state-dependence form $\sigma_{x, 2}$ to $\sigma_{z, 2}$. This block therefore displaces the motion entangled with the remaining population in the $|\downarrow\rangle$ state. The final $\hat{R}_{1}(\phi)$ pulse then creates the state $-e^{-i \phi}|\downarrow\rangle\left|\psi_{(+, \phi)}\right\rangle+$ $|a\rangle\left|\psi_{(-, \phi)}\right\rangle$, with $\left|\psi_{( \pm, \phi)}\right\rangle=\left(\mathbb{1} \pm e^{i \phi_{1}} \hat{\mathcal{D}}(\alpha)\right)\left|\psi_{\text {in }}\right\rangle$. We note that in the asymmetric implementation, the effective qubit is given by transition $1|\downarrow\rangle,|a\rangle$. The computational basis prior to fluorescence detection is swapped in this implementation by changing the last pulse phase $R_{1}(\phi)$ to $R_{1}(\phi+\pi)$ instead of adding an additional $\pi$ pulse.

\section{APPENDIX D: CALIBRATION OF MODULAR MEASUREMENTS}

We calibrate the SDF pulse and perform two additional laser phase calibrations. These calculations, together with automated calculations of phases due to Stark shifts using the known pulse durations and timings, allow us to run, in principle, arbitrarily long sequences of modular measurements.

\section{SDF pulse}

The SDF pulse is calibrated by first roughly balancing blue- and red-sideband powers and applying this to an initial ground-state cooled oscillator in the $|\downarrow\rangle$ internal level for a time $t_{\mathrm{SDF}}$. The decrease of $P(\downarrow)$ probability is observed, and iteratively, we find better balanced laser powers and a Stark shift of our transition by smoothing out the $P(\downarrow)$ signal at 0.5 probability for the time scales required in the experiment. From the calibrated SDF pulse, we extract the proportionality factor between pulse time and displacement size by fitting the qubit decay to its expected form $P(\downarrow)=\frac{1}{2}\left(1+e^{-2\left(c t_{\mathrm{SDF}}\right)^{2}}\right)$, with $c$ the floated proportionality constant. Typical values of $c$ obtained from these fits are $c \approx 0.035-0.028 \mu \mathrm{s}^{-1}$. An example of this calibration is given in Fig. 5(a).

\section{Laser-transition phase evolution}

To realize the modular measurements in the asymmetric implementation, we need the relative laser-transition phases, as well as the oscillator time evolution, to be phase locked. The SDF pulse addresses transition 2 with a bichromatic pulse, where the average of these two 

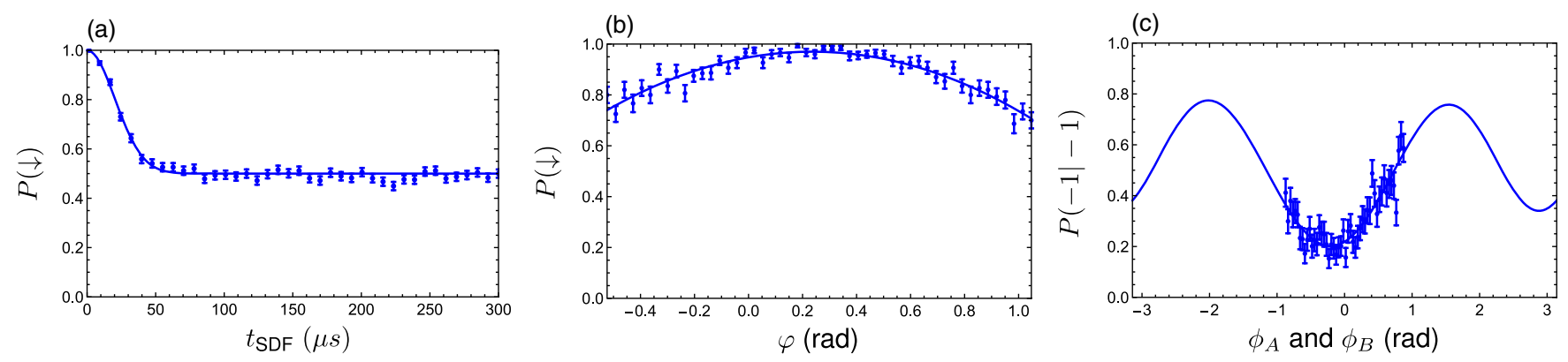

FIG. 5. Calibrations. Panel (a) shows a calibrated SDF pulse applied to a ground-state cooled ion and a fit to the expected behavior $P(\downarrow)=\frac{1}{2}\left(1+e^{-2\left(c t_{\mathrm{SDF}}\right)^{2}}\right)$. From this fit, we extract the proportionality constant $c$ between displacement size and $t_{\mathrm{SDF}}$, which we use in all analytic calculations of expected measurement results. Panel (b) shows the phase matching between addressing transition 2 with single frequency or with a bichromatic pulse. The sequence consists of a single $\pi / 2$ pulse followed by a SDF pulse, here with $t_{\mathrm{SDF}}=200 \mu \mathrm{s}$. Whenever the $\pi / 2$ pulse phase $\varphi$ is matched to the SDF, no superposition will be created; instead, the full wave packet is displaced, and therefore, $P(\downarrow) \equiv 1$ for any SDF duration. Finally, we need to calibrate the phase of the $R_{1}(\phi)$ pulse using an experimental calibration, as shown in panel (c). We use a sequence of two modular measurements with the same duration and opposite SDF directions in order to be able to observe a signal. We sweep both phases $\phi_{A}$ and $\phi_{B}$ simultaneously and read out the relevant minimum.

frequencies addresses transition 2 while the difference acts on the motional space: $\hat{H}_{\mathrm{SDF}}=[(\eta \hbar \Omega) / 2]\left(\hat{\sigma}_{+} e^{-i \bar{\phi}}+\right.$ $\left.\hat{\sigma}_{-} e^{i \bar{\phi}}\right)\left(\hat{a} e^{i \Delta \phi / 2+i \delta t}+\hat{a}^{\dagger} e^{-i \Delta \phi / 2-i \delta t}\right)$, with the phases $\bar{\phi}$ and $\Delta \phi$ given by the average and difference of the blue- and red-sideband laser phases. The average frequency when addressing transition 2 with the SDF differs by the calibrated Stark shift to the frequency used in a resonant carrier pulse. To account for this mismatch in phase evolution, we match the phase of the first $R_{2}$ rotation to the SDF and calculate the phases of the following $R_{2}(\varphi)$ pulses based on the calibrated Stark shift and the timing of our sequence. The action of the calibrated block $\hat{R}_{2}(0) \hat{\mathcal{D}}\left(\alpha(t) \hat{\sigma}_{x, 2}\right) \hat{R}_{2}(\pi)$ on $|\downarrow\rangle$ gives a constant probability $P(\downarrow) \equiv 1$ independent of the displacement size. An example using this characteristic for calibration is given in Fig. 5(b).

\section{Superposition phase controlled by $\boldsymbol{R}_{1}(\phi)$ pulse}

The phases of transition-1 pulses $R_{1}(\phi)$ are solely calibrated on the expected physics. We note that after each modular measurement, the qubit is detected and projected to $|\uparrow\rangle$. Therefore, in contrast to the oscillator, the lasertransition-1 and -2 phases are reset at the start of each modular measurement. Thus, for two sequential measurements with the same duration of the SDF pulse, the phase is identical. A single modular measurement with a general displacement $\alpha$ does not allow us to calibrate $\phi$. This is because, for large enough displacements, $P(\downarrow) \equiv 0.5$, which is independent of $\phi$. Instead, we calibrate the phase $\phi$ by a correlation measurement with settings $\alpha_{A}=-\alpha_{B}$, varying $\phi_{A}$ and $\phi_{B}$ jointly and fitting to the theoretical expectation. An example is given in Fig. 5(c).

\section{APPENDIX E: TIME SCALES OF EXPERIMENTAL SEQUENCE}

Cooling of the calcium ion is done by precooling $(1000 \mu \mathrm{s})$, Doppler cooling (500 $\mu \mathrm{s})$, electromagnetically induced transparency cooling $(400 \mu \mathrm{s})$, and, finally, resolved sideband cooling on the axial motional mode $(250 \mu \mathrm{s})$ to a mean occupation of about $\bar{n} \approx 0.05$ quanta. Note that $\pi / 2$ pulses on transition 2 take roughly $1.5 \mu \mathrm{s}$, while on transition 1, we need around $4 \mu \mathrm{s}$. Displacement operations take between 20 and $90 \mu \mathrm{s}$. Fluorescence detection takes $60 \mu \mathrm{s}$. The decision of the FPGA about whether to continue with the experiment or to restart the sequence takes $50 \mu \mathrm{s}$.

\section{APPENDIX F: COMPARISON OF THE TWO IMPLEMENTATIONS}

The symmetric implementation, besides its simpler pulse sequence, has several further advantages: The transition $|\downarrow\rangle,|\uparrow\rangle$ has approximately 6 times more laser power available and half the magnetic field sensitivity compared to qubits used in the asymmetric implementation $|\downarrow\rangle,|a\rangle$. Therefore, whenever possible, we use the symmetric implementation. Both implementations can be represented in the circuit model by the circuit given in Fig. 1.

\section{APPENDIX G: ANALYTIC CALCULATION OF SIGNALING IN TIME AND TIME CORRELATORS}

In order to calculate $S$ and $C$ for the symmetric as well as the asymmetric implementation, we first expand the expression for $P_{B}(b)$, which is identical for the two implementations because of their connection via a unitary, 
i.e., $\hat{U} \hat{F}_{ \pm}=\hat{E}_{ \pm}$, with $\hat{F}_{ \pm}(\phi, \alpha)=\frac{1}{2}\left(\mathbb{1} \pm e^{i \phi} \hat{\mathcal{D}}(\alpha)\right)$ the measurement operators of the asymmetric implementation, $\hat{E}_{ \pm}(\phi, \alpha)=\frac{1}{2}\left(\hat{\mathcal{D}}(-\alpha / 2) \pm e^{i \phi} \hat{\mathcal{D}}(\alpha / 2)\right)$ the measurement operators of the symmetric implementation, and $\hat{U}=\hat{\mathcal{D}}(-\alpha / 2)$. Note that

$$
\begin{aligned}
P_{B}(b) & =\left\langle\psi_{\text {in }}\left|\hat{E}_{b}^{\dagger} \hat{E}_{b}\right| \psi_{\text {in }}\right\rangle \\
& =\frac{1}{4}\left\langle\psi_{\text {in }}\left|2 \mathbb{1}+b e^{-i \phi_{B}} \hat{\mathcal{D}}\left(-\alpha_{B}\right)+b e^{i \phi_{B}} \hat{\mathcal{D}}\left(\alpha_{B}\right)\right| \psi_{\text {in }}\right\rangle \\
& =\frac{1}{2}\left\{1+b\left|m_{\alpha_{B}}\right| \cos \left(\phi_{B}+\arg \left(m_{\alpha_{B}}\right)\right),\right.
\end{aligned}
$$

where we defined the "displaced overlap" $m_{\alpha}$ and used its polar representation. Here, $m_{\alpha}=\left\langle\psi_{\text {in }}|\hat{\mathcal{D}}(\alpha)| \psi_{\text {in }}\right\rangle=$ $\left|m_{\alpha}\right| e^{i \arg \left(m_{\alpha}\right)}$. Further, we expand $P_{B A}(b, a)$, which depends on the post-measurement state of $A$ and thus on the implementation we choose:

$$
P_{B A}(b, a)=\left\langle\psi_{\text {in }}\left|\hat{F}_{a}^{\dagger} \hat{U}^{\dagger} \hat{F}_{b}^{\dagger} \hat{F}_{b} \hat{U} \hat{F}_{a}\right| \psi_{\text {in }}\right\rangle .
$$

Defining the geometric phase as $\Phi=\operatorname{Im}\left(\alpha_{B} \alpha_{A}^{*}\right)$, we find, for the symmetric $P$ and asymmetric $\tilde{P}$ implementations,

$$
\begin{aligned}
\tilde{P}_{B A}(b, a)= & \frac{1}{16}\left(4+\left\langle\psi_{\text {in }}\right|\left(b \hat{\mathcal{D}}\left(-\alpha_{B}\right) e^{-i \phi_{B}}\left(1+e^{-2 i \Phi}\right)+b \hat{\mathcal{D}}\left(\alpha_{B}\right) e^{i \phi_{B}}\left(1+e^{2 i \Phi}\right)+2 a\left(\hat{\mathcal{D}}\left(-\alpha_{A}\right) e^{-i \phi_{A}}+\hat{\mathcal{D}}\left(\alpha_{A}\right) e^{i \phi_{A}}\right)\right.\right. \\
& +a b \hat{\mathcal{D}}\left(\alpha_{A}-\alpha_{B}\right) e^{i\left(\phi_{A}-\phi_{B}-\Phi\right)}+a b \hat{\mathcal{D}}\left(\alpha_{A}+\alpha_{B}\right) e^{i\left(\phi_{A}+\phi_{B}+\Phi\right)}+a b \hat{\mathcal{D}}\left(-\alpha_{A}-\alpha_{B}\right) e^{i\left(-\phi_{A}-\phi_{B}-\Phi\right)} \\
& \left.\left.+a b \hat{\mathcal{D}}\left(-\alpha_{A}+\alpha_{B}\right) e^{i\left(\phi_{B}-\phi_{A}+\Phi\right)}\right)\left|\psi_{\text {in }}\right\rangle\right), \\
P_{B A}(b, a)= & \frac{1}{16}\left(4+\left\langle\psi_{\text {in }}\right|\left(b \left(\hat{\mathcal{D}}\left(-\alpha_{B}\right) e^{-i \phi_{B}}\left(e^{i \Phi}+e^{-i \Phi}\right)+b\left(\hat{\mathcal{D}}\left(\alpha_{B}\right) e^{i \phi_{B}}\left(e^{i \Phi}+e^{-i \Phi}\right)+2 a\left(\hat{\mathcal{D}}\left(-\alpha_{A}\right) e^{-i \phi_{A}}+\hat{\mathcal{D}}\left(\alpha_{A}\right) e^{i \phi_{A}}\right)\right.\right.\right.\right. \\
& +a b \hat{\mathcal{D}}\left(\alpha_{A}-\alpha_{B}\right) e^{i\left(\phi_{A}-\phi_{B}\right)}+a b \hat{\mathcal{D}}\left(\alpha_{A}+\alpha_{B}\right) e^{i\left(\phi_{A}+\phi_{B}\right)}+a b \hat{\mathcal{D}}\left(-\alpha_{A}-\alpha_{B}\right) e^{i\left(-\phi_{A}-\phi_{B}\right)} \\
& \left.\left.+a b \hat{\mathcal{D}}\left(-\alpha_{A}+\alpha_{B}\right) e^{i\left(\phi_{B}-\phi_{A}\right)}\right)\left|\psi_{\text {in }}\right\rangle\right) .
\end{aligned}
$$

The dependence on the measurement outcomes $a, b$ is of the form:

$$
P_{B A}(b, a)=a c_{1}+b c_{2}+a b c_{3}+c_{4} .
$$

Using this result, we find

$$
\begin{aligned}
C_{A B} & =\sum_{a, b} a b P_{B A}(b, a)=4 c_{3} \\
S & =P_{B}(b=+1)-P_{B(A)}(b=+1) \\
& =P_{B}(b=+1)-\sum_{a} P_{B A}(b, a) \\
& =P_{B}(b=+1)-2 c_{2}-2 c_{4},
\end{aligned}
$$

from which the formulas of the main text follow. The analogous is true for $\tilde{P}_{B A}(b, a)$, from which $\tilde{C}_{A B}$ and $\tilde{S}_{A B}$ follow.

\section{APPENDIX H: ANALYTIC CALCULATION OF MEASUREMENT PROBABILITIES}

The analytic calculation is based on a few basic principles, which can be used for any number and implementation of modular measurements with a wide variety of input states, including mixed thermal states. We automated these few principles using Mathematica, and they are summarized in the following. First, we note that by combining displacement operators using $\hat{\mathcal{D}}(\beta) \hat{\mathcal{D}}(\alpha)=$ $e^{i \operatorname{Im}\left(\beta \alpha^{*}\right)} \hat{\mathcal{D}}(\alpha+\beta)$, any measurement probability can be expressed as a summation over terms of the same type. This is shown in the formula below, where we additionally use the cyclicity of the trace $(\operatorname{Tr}(A B C)=\operatorname{Tr}(C A B))$, that $\hat{\mathcal{D}}^{\dagger}(\gamma)=\hat{\mathcal{D}}(-\gamma)$ and evaluate the probability in the Fock state basis.

$$
\begin{aligned}
P_{A}(a) & =\operatorname{Tr}\left\{\hat{E}_{a}^{\dagger}(\phi, \alpha) \hat{E}_{a}(\phi, \alpha) \hat{\rho}_{\text {in }}\right\} \\
& =\sum_{i, j} a_{i, j} \operatorname{Tr}\left\{\hat{\mathcal{D}}\left(\beta_{i}\right) \hat{\rho}_{0} \hat{\mathcal{D}}^{\dagger}\left(\gamma_{j}\right)\right\} \\
& =\sum_{i, j} a_{i, j} e^{-i \operatorname{Im}\left(\gamma_{j} \beta_{i}^{*}\right)} \sum_{n=0}^{\infty}\left\langle n\left|\hat{\mathcal{D}}\left(\delta_{i j}\right) \hat{\rho}_{0}\right| n\right\rangle .
\end{aligned}
$$

The measurement input state $\hat{\rho}_{\text {in }}$ can be the post-measurement state of previous experiments. Thus, we inserted $\hat{\rho}_{\text {in }}=\sum_{i, j} a_{i, j} \hat{\mathcal{D}}\left(\beta_{i}\right) \hat{\rho}_{0} \hat{\mathcal{D}}^{\dagger}\left(\gamma_{j}\right)$, with $a_{i, j} \in \mathbb{C}$ and $\hat{\rho}_{0}$ the input state for the first measurement. Further, we define $\delta_{i j}=\beta_{i}-\gamma_{j}$. For the states considered in this paper, $\hat{\rho}_{0}$ is given by either (i) ground state $|0\rangle\langle 0|$, (ii) first excited state $|1\rangle\langle 1|$, (iii) thermal state $\hat{\rho}_{\text {th }}=\sum_{k=0}^{\infty}\left[\bar{n}^{k} /(1+\bar{n})^{k+1}\right]|k\rangle\langle k|$, or (iv) squeezed vacuum state $\hat{S}(\xi)|0\rangle$. For states (i)-(iii), the probabilities can be evaluated using $\langle n|\hat{\mathcal{D}}(\alpha)| n\rangle=$ $e^{-\frac{1}{2}|\alpha|^{2}} L_{n}^{0}\left(|\alpha|^{2}\right)$, where for (iii) the additional thermal sum is calculated with the relation $\sum_{n=0}^{\infty} L_{n}^{\lambda}(z) w^{n}=$ $(1-w)^{-\lambda-1} e^{[(w z) /(w-1)]},|w|<1$. Here, $L$ denotes the Laguerre polynomials. For the squeezed vacuum state $\left[\hat{S}(\xi)=e^{\left(\xi \hat{a}^{\dagger}-\xi^{*} \hat{a}^{2}\right) / 2}\right.$ with $\left.\xi=r e^{i \phi}\right]$, we evaluate the trace in the corresponding squeezed Fock state basis 
and interchange squeezing and displacement operators with the relation $\hat{S}(\xi) \hat{\mathcal{D}}\left(\beta^{\prime}\right)|0\rangle=\hat{\mathcal{D}}(\beta) \hat{S}(\xi)|0\rangle$, with $\beta^{\prime}=\cosh (r) \beta-e^{i \phi} \sinh (r) \beta^{*}$,

$$
\begin{aligned}
P_{A}(a) & =\sum_{i, j} a_{i, j} e^{-i \operatorname{Im}\left(\gamma_{j} \beta_{i}^{*}\right)} \sum_{n=0}^{\infty}\left\langle n\left|\hat{S}^{\dagger}(\xi) \hat{\mathcal{D}}\left(\delta_{i j}\right) \hat{S}(\xi)\right| 0\right\rangle\langle 0 \mid n\rangle \\
& =\sum_{i, j} a_{i, j} e^{-i \operatorname{Im}\left(\gamma_{j} \beta_{i}^{*}\right)} \sum_{n=0}^{\infty}\left\langle n\left|\hat{\mathcal{D}}\left(\delta_{i j}^{\prime}\right)\right| n\right\rangle .
\end{aligned}
$$

\section{APPENDIX I: NSIT, KRAUS OPERATORS, AND COMMUTATORS}

If the order of measurements $A, B$ does not matter, then for projective measurements, their respective observables commute. For the generalized measurements considered in here, independence of the measurement order does not imply that the corresponding observables commute, which can be seen through the following argument. Still,

there exists a general relation between Kraus operators and NSIT: $\left\langle\left[\hat{E}_{A}^{a}, \hat{E}_{B}^{\dagger b} \hat{E}_{B}^{b}\right]\right\rangle=0 \Rightarrow$ NSIT. This relation is derived here:

$P_{B(A)}(b)=\sum_{a} P_{B A}(b, a)=\sum_{a} \operatorname{Tr}\left\{\hat{E}_{a}^{\dagger A} \hat{E}_{b}^{\dagger B} \hat{E}_{b}^{B} \hat{E}_{a}^{A} \hat{\rho}_{i}\right\}$

Using $\left\langle\left[\hat{E}_{a}^{A}, \hat{E}_{b}^{\dagger B} \hat{E}_{b}^{B}\right]\right\rangle=0$, this translates into

$$
\begin{aligned}
P_{B(A)}(b) & =\sum_{a} \operatorname{Tr}\left\{\hat{E}_{a}^{\dagger A} \hat{E}_{a}^{A} \hat{E}_{b}^{\dagger B} \hat{E}_{b}^{B} \hat{\rho}_{i}\right\} \\
& =\operatorname{Tr}\left\{\sum_{a} \hat{E}_{a}^{\dagger A} \hat{E}_{a}^{A} \hat{E}_{b}^{\dagger B} \hat{E}_{b}^{B} \hat{\rho}_{i}\right\} \\
& =\operatorname{Tr}\left\{\hat{E}_{b}^{\dagger B} \hat{E}_{b}^{B} \hat{\rho}_{i}\right\}=P_{B}(b) .
\end{aligned}
$$

Straightforward calculation of the commutator $\left[\hat{E}_{A}^{a}, \hat{E}_{B}^{\dagger b} \hat{E}_{B}^{b}\right]$ for the two implementations, as well as the commutator of the modular observables $\left[\hat{Q}_{A}, \hat{Q}_{B}\right]$, leads to the

(c)

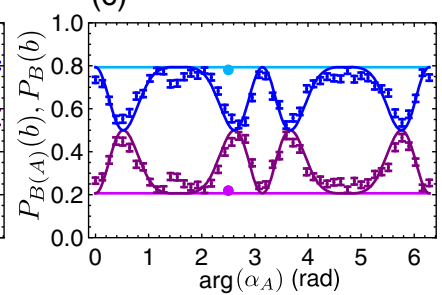

(d)

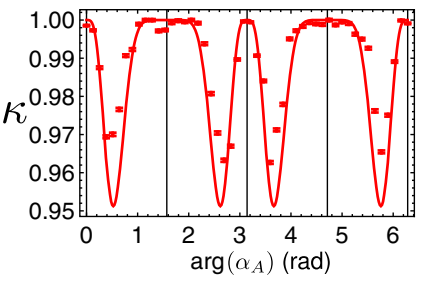

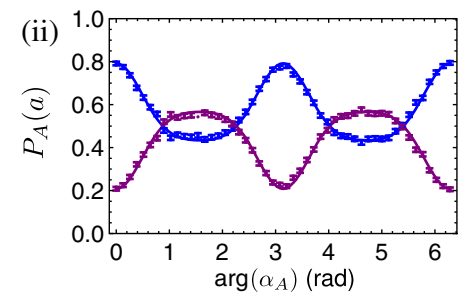
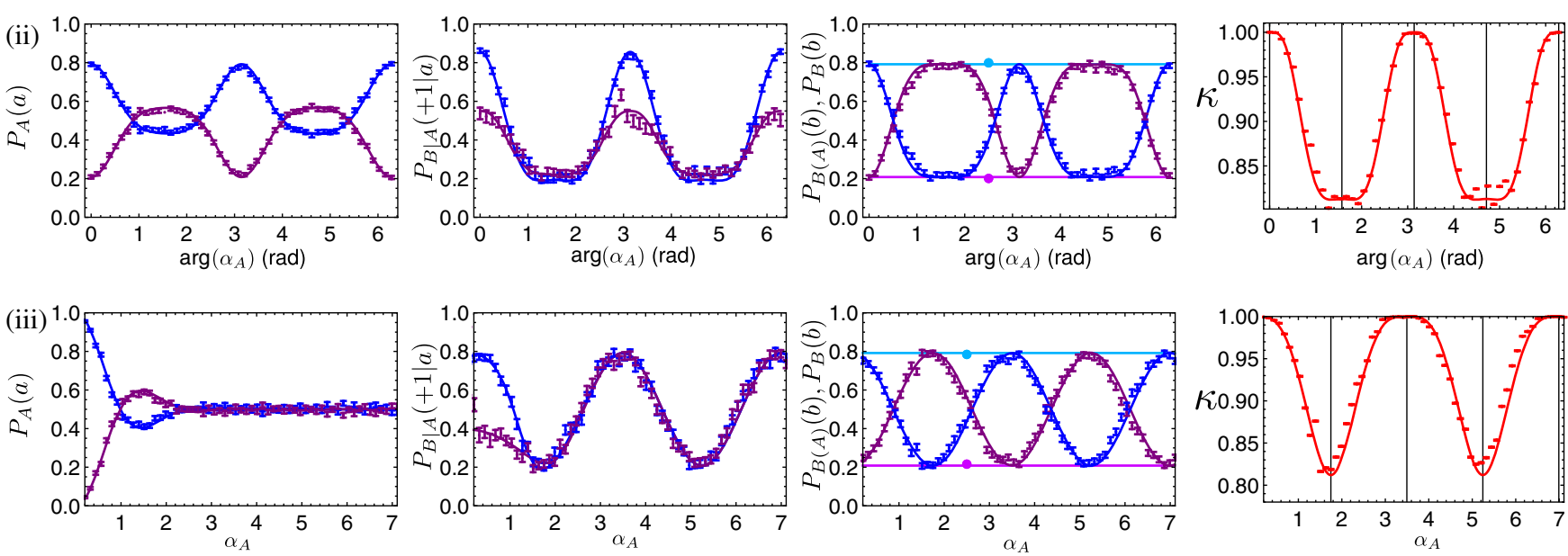

FIG. 6. Additional SIT and NSIT data compared to observable commutation. Columns (a) and (b) show the direct-detection data obtained by first measuring $A$ and then $B$. The blue line corresponds to $a=+1$, while purple stands for $a=-1$. Column (c) shows the result of measuring $B$ alone. Here, $P_{B}$ is given as a single point plotted at $\arg \left(\alpha_{A}\right)=2.5$, and it is compared to $P_{B(A)}$, which is calculated from the results of columns (a) and (b). The blue line is for $b=+1$, while purple is for $b=-1$. Finally, the classical fidelity $\kappa \equiv \sum_{b} \sqrt{P_{B}(b) P_{B(A)}(b)}$ [35] is shown in column (d). The classical fidelity can be used as an alternative to $S$ in order to quantify the amount of SIT. For NSIT measurements, $\kappa=1$. The black vertical lines in column (d) indicate settings for which the underlying observables commute. (i) Asymmetric implementation, SIT as a function of $\arg \left(\alpha_{A}\right)$, with $\left|\alpha_{A}\right|=\sqrt{\pi}, \alpha_{I}=\sqrt{\pi}, \alpha_{B}=-\sqrt{\pi}$, $\phi_{A}=\phi_{B}=0$. The data vary between SIT and NSIT, where NSIT is observed for the settings where the observables commute. (ii) The same experiment with the symmetric implementation, $\phi_{A}=\phi_{B}=\pi$. We observe that this implementation can be SIT even in cases where the observables commute. (iii) SIT as a function of $\alpha_{A}$ in the symmetric implementation with $\alpha_{B}=\alpha_{I} \approx \sqrt{\pi}$. Again, SIT is observed at points where the observables commute. 
conditions for NSIT or commutation of the observables given by

Symmetric: $\operatorname{Im}\left(\alpha_{B} \alpha_{A}^{*}\right)=2 \pi k_{1}, \quad k_{1} \in \mathbb{Z} \Rightarrow$ NSIT,

Asymmetric: $\operatorname{Im}\left(\alpha_{B} \alpha_{A}^{*}\right)=\pi k_{2}, \quad k_{2} \in \mathbb{Z} \Rightarrow$ NSIT,

$$
\left[\hat{Q}_{A}, \hat{Q}_{B}\right]: \operatorname{Im}\left(\alpha_{B} \alpha_{A}^{*}\right)=\pi k_{3}, \quad k_{3} \in \mathbb{Z} \Rightarrow\left[\hat{Q}_{A}, \hat{Q}_{B}\right]=0 .
$$

Therefore, if the commutator of the observables vanishes with an odd $k_{3}$ number, then $\operatorname{Im}\left(\alpha_{B} \alpha_{A}^{*}\right)=\pi k_{\text {odd }} \neq 2 \pi k_{1}$; thus, the symmetric implementation is SIT. This is a general case for which the observables commute but the sequential measurements are SIT. In Fig. 6, additional data for SIT and NSIT experimental sequences are shown. In particular, one can compare the two different implementations and see that NSIT does not imply commutation of the observables.

\section{APPENDIX J: THEORETICAL WIGNER FUNCTION PLOTS OF EXPERIMENTALLY CREATED STATES}

In Fig. 3(d) of the main text, we plot the Wigner functions of the states created during our measurement of NSIT for modular position and momentum for one example of input state. The chosen state was a superposition of a displaced ground-state cooled oscillator. The same experiment was also performed with either an oscillator in a squeezed state or in the first excited state. In Fig. 7, an equivalent plot with the same orientation of initial superposition, but now based on a (a) first excited or (b) squeezed state, is shown.

(a)
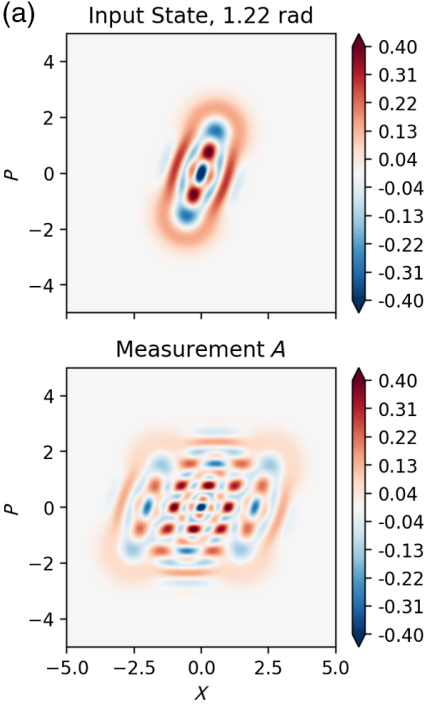
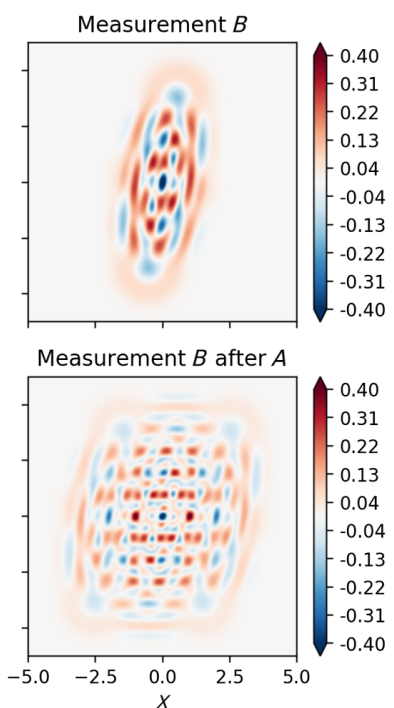

APPENDIX K: CORRELATOR FULL DATA SET

The full measurement data of the correlation measurements presented in Fig. 4(a) of the main text are shown in Fig. 8. Besides the measured correlators, the probabilities measured in the first measurement $A$ and the second measurement $B$ are also shown.

\section{APPENDIX L: RAMSEY ANALOGY AND ADVANTAGE OF SEQUENTIAL MEASUREMENTS}

The modular measurements presented can be viewed as Ramsey measurements coupling to a quantum field, which is given by the oscillator phase space. Thus, the best semiclassical comparison is given by a Ramsey measurement coupling to a classical variable $x(t)$. Therefore, we compare Ramsey spin measurements with both a semiclassical $\hat{H} \propto|\uparrow\rangle\langle\uparrow| x(t)$ and full quantum Hamiltonian $\hat{H} \propto|\uparrow\rangle\langle\uparrow|\left(\hat{a} e^{i \Delta \phi / 2}+\hat{a}^{\dagger} e^{-i \Delta \phi / 2}\right)$. An extensive discussion of this comparison can be found in the supplemental material of Ref. [8].

In previous experiments with superposition states, single detection results were used to confirm the creation of superposition states $[25,28,29]$. Such single-time detection results could, in principle, emerge from the coupling to a classical variable $x(t)$. In particular, if $x(t)$ contains dominant frequency components, a variety of oscillations and revivals in the qubit probabilities can be observed. As an illustration, we consider a simple example: $x(t)=A \cos (2 \pi f t)$ is given by a single frequency component $f$ with a fluctuating amplitude $A$. The amplitude fluctuates on slow time scales compared to a single experimental shot, and its probability distribution is given by the Gaussian $P(A)=[1 /(\sigma \sqrt{2 \pi})] e^{\left.-\frac{1}{2}\left(A-A_{0}\right) / \sigma\right]^{2}}$; the
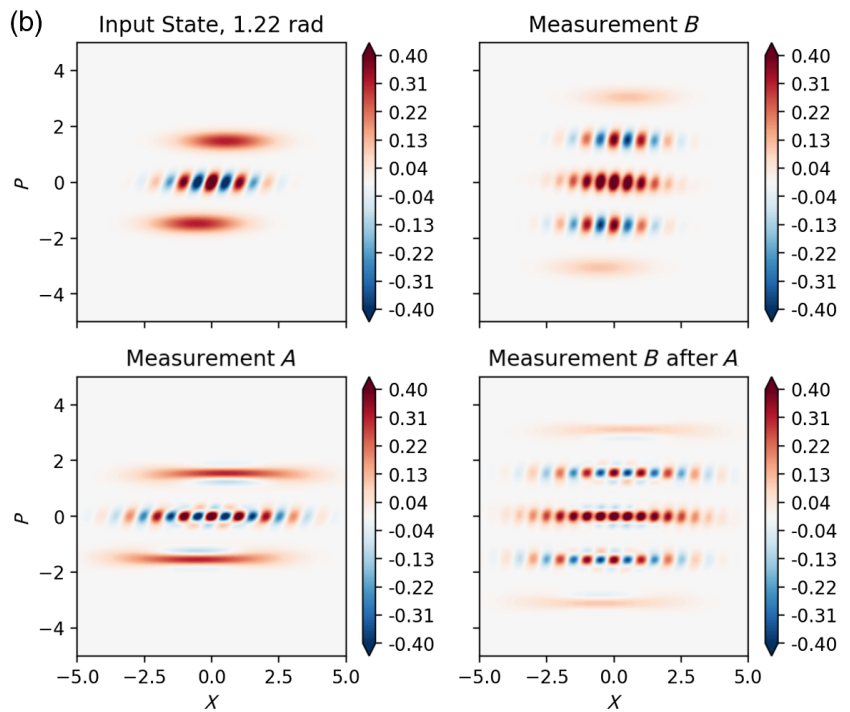

FIG. 7. Theoretical Wigner function plots of two more examples of input states for the measurements presented in Fig. 3 of the main text. The displayed input states are $\left(\hat{\mathcal{D}}\left(-\left|\alpha_{B}\right| e^{i \phi_{I}} / 2\right)+\hat{\mathcal{D}}\left(\left|\alpha_{B}\right| e^{i \phi_{I}} / 2\right)\right)|\phi\rangle$ with $\phi_{I}=1.22 \operatorname{rad}$ and (a) $|\phi\rangle=|1\rangle$ and (b) $\hat{S}(-0.82)|0\rangle$. Further, their post-measurement states with the result +1 during the experimental sequence are shown. 


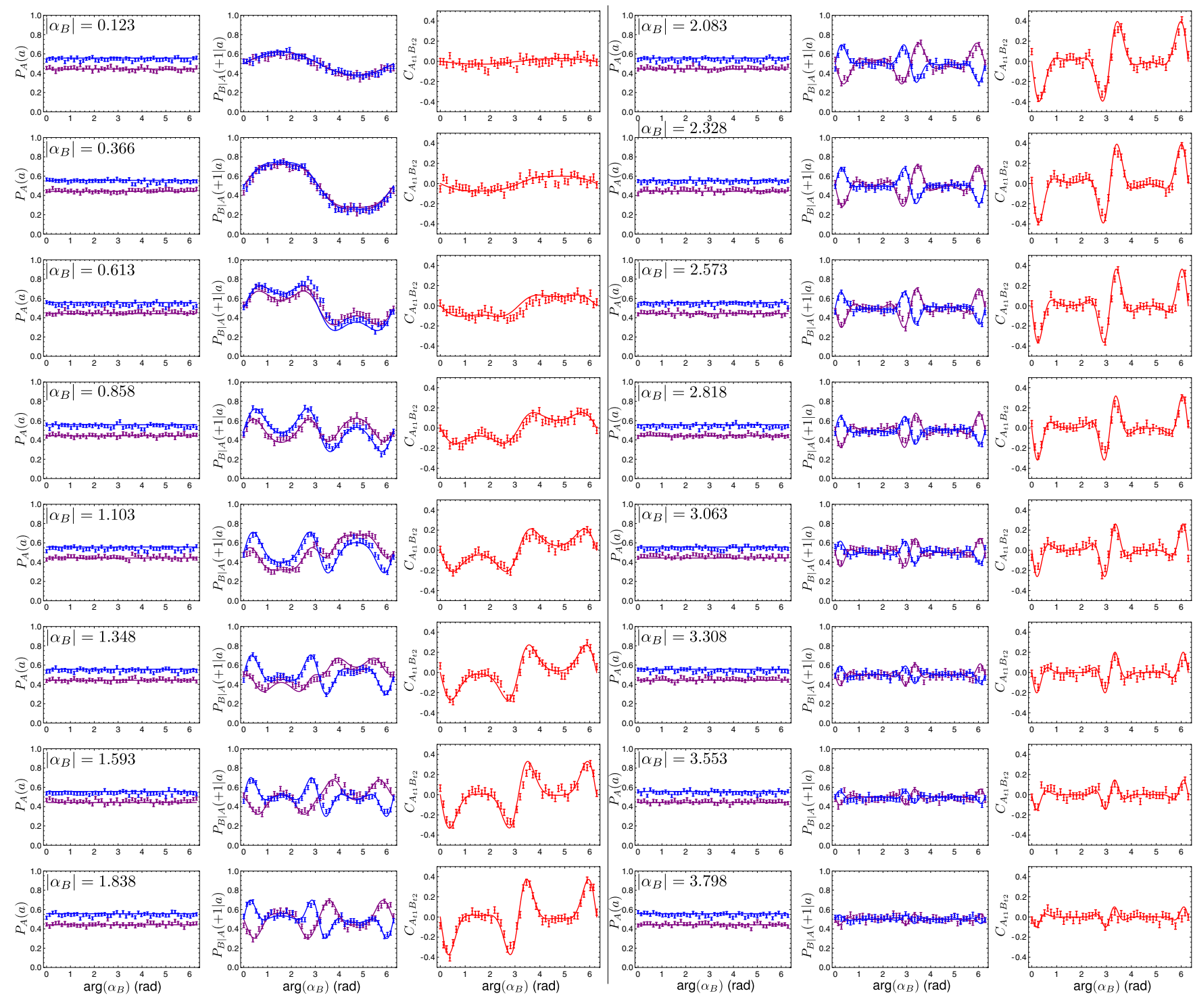

FIG. 8. Two-time correlation measurement in the asymmetric implementation. The fixed experimental settings were $\alpha_{A}=2.1$, $\phi_{A}=0, \phi_{B}=\pi / 2$. The blue points show $a=+1$ measurement results, while purple points shows $a=-1$. Solid lines show the expectations for an ideal experiment. Error bars of $P_{A}(a), P_{B \mid A}(+1 \mid a)$ are given as SEM, from these the error bars of $C_{A B}$ are propagated. The data sets shown here together create the false-color plot of Fig. 4(a) in the main text.

experiment is synchronized with respect to the noise frequency (an example of such a noise source would be noise due to the main lines measured by a line-triggered experiment). For such a periodic noise source, we find $\langle\hat{Q}\rangle=\langle P(+1)-P(-1)\rangle=$ $-e^{\frac{1}{2}\{[\sin (2 \pi f T) \sigma] / 2 \pi f\}} \cos \left(\phi+\left[A_{0} /(2 \pi f)\right] \sin (2 \pi f T)\right)$, where $T$ is given by the Ramsey interaction time and $\phi$ is the phase of the second Ramsey pulse. Note that $\langle\hat{Q}\rangle$ is plotted in Fig. 9, where we can see that it exhibits very similar oscillations to those observed in experiments like in Ref. [28]. Single measurements thus have a hard time proving that the experiments actually create Schrödinger's cat-like superpositions. Nature could be malicious, and one could just couple to a classical variable $x(t)$, giving rise to the observed oscillations and revivals.
The distinction between the coupling to a classical field or a quantum field is easier when considering sequential measurements. In the quantum case, the first measurement can create a superposition state of the quantum field, which can change the statistics of the second measurement (SIT). In the classical case, the variable $x(t)$ is not changed by the first measurement, and SIT will not be observed between two measurements.

\section{APPENDIX M: LEGGETT-GARG VIOLATION MEASUREMENT SETTINGS}

In order to find the measurement settings with which we violate the LGI, we calculate the analytic expression for the value of $L$ depending on the initial ion temperature 
(a)

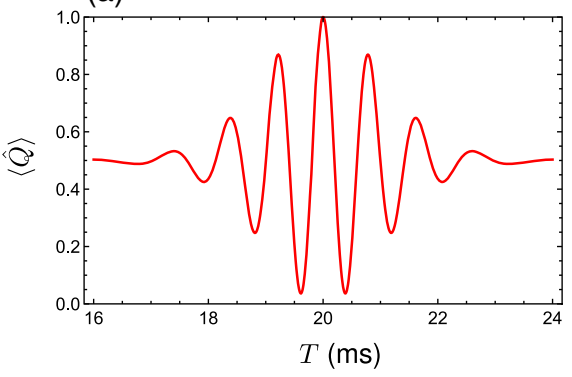

(b)

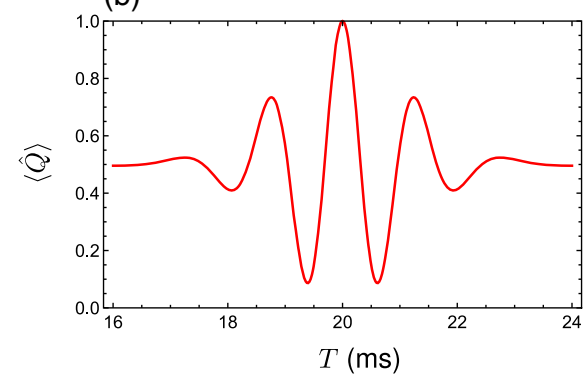

(c)

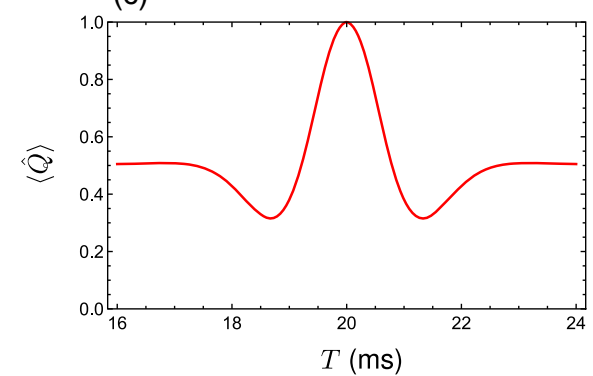

FIG. 9. Expected results for a Ramsey measurement coupling to $x(t)=A \cos (2 \pi f t)$, a classical single-frequency noise source. The amplitude $A$ of this single-frequency noise fluctuates on time scales slower than an experimental shot with a Gaussian probability distribution $P(A)=[1 /(\sigma \sqrt{2 \pi})] e^{-\frac{1}{2}\left[\left(A-A_{0}\right) / \sigma\right]^{2}}$. The coupling constant is assumed to be $1: \hat{H}=|\uparrow\rangle\langle\uparrow| x(t)$. Thus, we find $\langle\hat{Q}\rangle=\langle P(+1)-P(-1)\rangle=-e^{\frac{1}{2}\{[\sin (2 \pi f T) \sigma] / 2 \pi f\}} \cos \left(\phi+\left[A_{0} /(2 \pi f)\right] \sin (2 \pi f T)\right)$, with $T$ given by the Ramsey wait time and $\phi$ the second Ramsey pulse phase. We show three different amplitudes $A_{0}$ : (a) $A_{0}=8000$, (b) $A_{0}=5000$, and (c) $A_{0}=2000$, of the noise, with the noise frequency fixed to $50 \mathrm{~Hz}, \sigma=1000, \phi=0$. The oscillations resemble characteristic traces of Schrödinger cat states, such as those found in Ref. [28].

and the displacement size $\alpha$, as well as the measurement settings-A: $\left(\theta_{1}, \phi_{A}\right), B:\left(\theta_{2}, \phi_{B}\right), C:\left(\theta_{3}, \phi_{C}\right)$. For each temperature and displacement $\alpha$, we maximize the analytic expression over $\theta_{1}, \theta_{2}, \theta_{3}$ and $\phi_{A}, \phi_{B}, \phi_{C}$ using Mathematica. To do so, we first find a local maximum for a small displacement $\alpha=0.2$; then, we use the settings found from this analysis as an initial guess for the maximization for a slightly larger displacement $\alpha=0.25$; in this way, we successively find the settings for larger displacements. In Fig. 10, some raw data of $L$ violation measurements, together with the experimental settings used, are shown.

The temperature of the oscillator is calibrated by shortening the cooling sequence used and subsequently reading out the Fock state populations of the oscillator, fitting them to a thermal state of the oscillator. Before each LeggettGarg experiment, the phase $\phi$ is calibrated for the displacement size and temperature in the manner described before. Based on this single calibration, the three correlations are measured.

\section{APPENDIX N: EFFECT OF NOISE ON LEGGETT-GARG INEQUALITY VIOLATIONS}

The motional dephasing is accounted for by solving the Lindblad master equation during the state-dependent-force pulses with a dephasing operator $\sqrt{30}\left(\hat{a} \hat{a}^{\dagger}+\hat{a}^{\dagger} \hat{a}\right)$ with 30 dephasing jumps/s. The linewidth of the transition $k=1$ is known from Ramsey measurements to be $l \approx 665 \mathrm{~Hz}$ FWHM and varies on time scales longer than an experimental shot; thus, we include it by averaging over 4000 randomly chosen phases $\phi$ from a normal distribution with $\sigma=\left[l \pi t_{\mathrm{SDF}} /(\sqrt{2 \ln (2)})\right]+0.087$. The last term accounts for phase calibration errors.

\section{APPENDIX O: PENALIZED LGI}

The paper by Kujala et al. [32] considers cyclic contextuality inequalities. Some LGIs, for example, $\tilde{C}_{A B}+\tilde{C}_{B C}+\tilde{C}_{C A}<1$, are special cases of these cyclic contextuality inequalities. The paper addresses the problem that even if an experimenter intends to perform compatible measurements because of experimental fluctuations and imprecisions, there will still be a certain amount of SIT between the sequential measurements performed. The work derives penalized contextuality bounds to account for these imprecisions. The derived penalization is expressed in the notation of this work as $T S=2\left(\left|\tilde{S}_{A B}\right|+\right.$ $\left.\left|\tilde{S}_{B C}\right|+\left|\tilde{S}_{C A}\right|\right)$ and can be interpreted as the total amount of SIT observed. They consider cyclic measurements; thus, each measurement is performed once as the first measurement in the sequence and once as the second measurement. This allows the penalization to be extracted directly from the contextuality bound measurements.

In contrast, the inequality we considered in this work is not cyclic, $C_{A B}+C_{B C}-C_{A C}<1$. Only $B$ is performed once as a first measurement and another time as a second measurement. Thus our LGI violation measurements only allow the extraction of $\tilde{S}_{A B}$. Performing an additional measurement of $C$ directly on the input state would allow us to extract $\left|\tilde{S}_{B C}\right|$ and $\left|\tilde{S}_{A C}\right|$ and then to calculate a penalized $L$ value: $L_{\text {pen }}=L-2\left(\left|\tilde{S}_{A B}\right|+\left|\tilde{S}_{B C}\right|+\left|\tilde{S}_{A C}\right|\right)=L-T S$.

In this penalization, we assume that there is no backward SIT. This means that if we first perform a measurement $A$ and then $B$, we obtain $P_{A}(a)=$ $P_{(B) A}(a) \equiv \sum_{b} P_{B A}(b, a)$, which, in a real experiment, will only be approximately given. The penalization for the cyclic inequalities also contains this type of fluctuation to some extent. Further, there might be subtleties that we miss at this stage. 

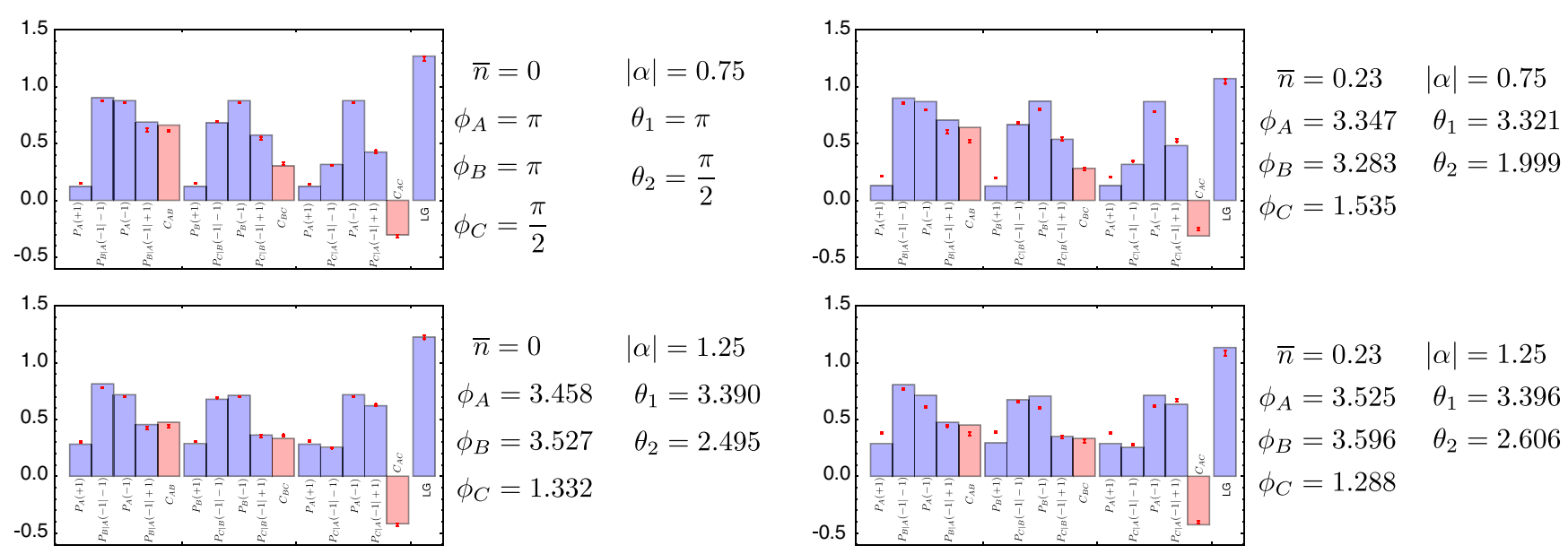

$$
\begin{aligned}
\bar{n} & =0 & |\alpha| & =1.25 \\
\phi_{A} & =3.458 & \theta_{1} & =3.390 \\
\phi_{B} & =3.527 & \theta_{2} & =2.495 \\
\phi_{C} & =1.332 & &
\end{aligned}
$$

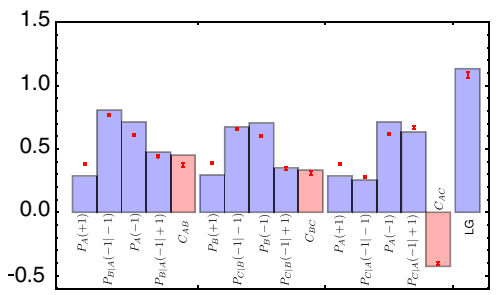

$$
\bar{n}=0.23 \quad|\alpha|=1.25
$$

$\phi_{A}=3.525 \quad \theta_{1}=3.396$

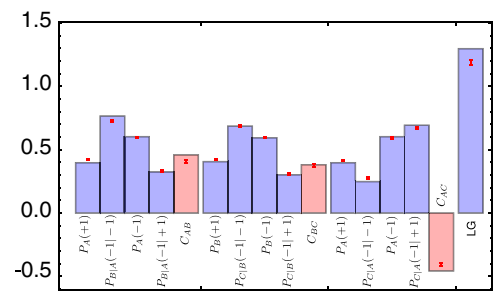

$$
\begin{aligned}
\bar{n} & =0 & & |\alpha|=1.75 \\
\phi_{A} & =3.459 & & \theta_{1}=3.331 \\
\phi_{B} & =3.656 & & \theta_{2}=2.756
\end{aligned}
$$

$\phi_{C}=1.171$

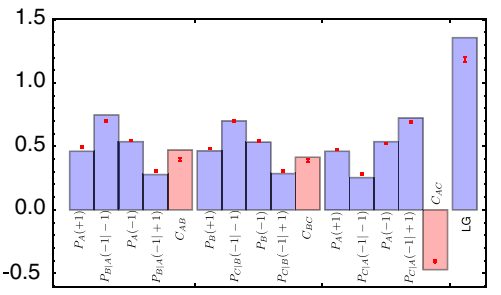

$$
\begin{aligned}
\bar{n} & =0 & |\alpha| & =2.25 \\
\phi_{A} & =3.433 & \theta_{1} & =3.270 \\
\phi_{B} & =3.676 & & \theta_{2}=2.885
\end{aligned}
$$

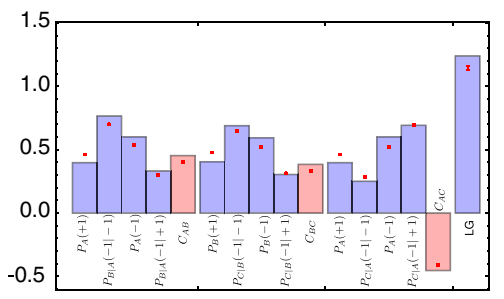

$\phi_{B}=3.596$

$\theta_{2}=2.606$

$\phi_{C}=1.118$

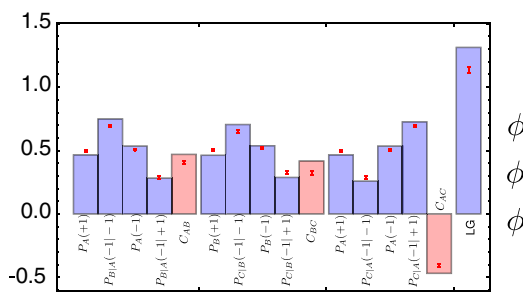

$$
\bar{n}=0.23
$$

$|\alpha|=2.25$

$\phi_{A}=3.652 \quad \theta_{1}=3.261$

$\phi_{B}=3.467 \quad \theta_{2}=2.903$

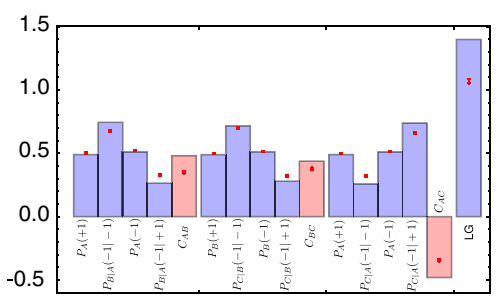

$$
\bar{n}=0 \quad|\alpha|=2.75
$$

$\phi_{A}=3.618 \quad \theta_{1}=3.232$

$\phi_{B}=3.481 \quad \theta_{2}=2.960$

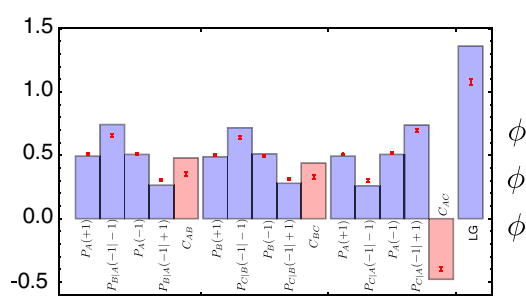

$\bar{n}=0.23 \quad|\alpha|=2.75$

$\phi_{A}=4.055 \quad \theta_{1}=3.228$

$\phi_{C}=1.291$

FIG. 10. A sample of individual detection data and measurement settings for the Leggett-Garg violations presented in Fig. 4(b) of the main text. The data are from the set $\bar{n} \approx 0$ and $\bar{n} \approx 0.23$. Data are shown as red points, while the expectations for an ideal experiment are shown as bars. Blue bars are the detection data, and the three red bars show the correlations calculated from these detections. We see qualitatively good agreement for smaller $\alpha$, which decreases for higher displacements, mainly due to dephasing noise in the experimental system. The settings $\theta_{1}$ and $\theta_{2}$ correspond to $\theta_{1}=\theta_{B}-\theta_{A}, \theta_{2}=\theta_{C}-\theta_{B}$.

Our LGI protocol is based on performing incompatible measurements; thus, we expect SIT between the measurements. Analytic calculation of the amount of built-in SIT in our protocol [see Fig. 11(a)] shows that this is indeed the case for displacements of around $\alpha=1$. But the amount of SIT approaches zero for the larger displacements. From this result, we conclude that for large displacements, the ideal protocol approaches $L=1.5$ with NSIT measurements at the two-time level. If we subtract the theoretical amount of built-in SIT from our data [see Fig. 11(b)], then at our experimentally achieved size of displacements, some points violate the LGI in this penalized fashion. These are the points highlighted with diamonds in the main part of the paper Fig. 4(b). Further, we can check how close our experiment resembles the theoretical amount of built-in SIT by extracting $\left|\tilde{S}_{A B}\right|$ from our experimental data [see Fig. 11(c)]. The theoretical expectation for $\left|\tilde{S}_{A B}\right|$ is around zero and never exceeds 0.02. The amount of SIT we measure is close to zero but slightly higher than this theoretical 
(a)

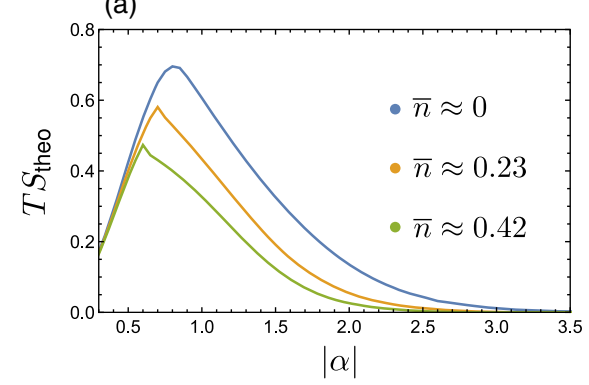

(b)

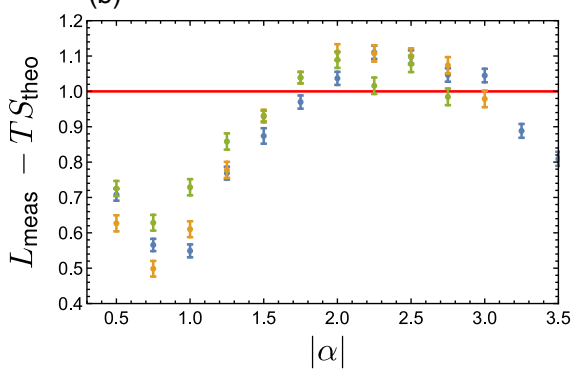

(c)

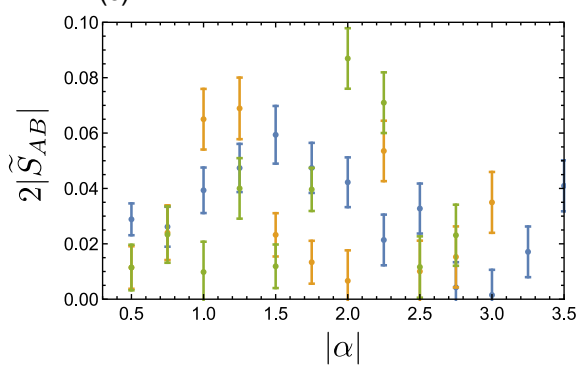

FIG. 11. Penalization of $L$ for built-in two-time SIT. (a) Analytic calculation of the total amount of SIT $T S=2\left(\left|\tilde{S}_{A B}\right|+\left|\tilde{S}_{B C}\right|+\left|\tilde{S}_{A C}\right|\right)$ due to incompatible settings used to violate the LGI. The TS approaches zero for the larger displacement $\alpha$. (b) The measured data penalized by the theoretical amount of built-in SIT. Several points around $\alpha=2.25$ are still able to violate the LGI in this penalized fashion. (c) $\tilde{S}_{A B}$ extracted from the experimental data. The amount of SIT is higher than expected from the analytic calculation, which predicts values up to 0.02 . But the values stay close to zero. Note that the total SIT is dominated by $\left|\tilde{S}_{B C}\right|$ and $\left|\tilde{S}_{A C}\right|$.

expectation. The higher amount of SIT is expected given the accuracy with which we can calibrate and perform our experiments. A number of methods have been proposed for performing a LGI test using NSIT measurements; see, for example, Ref. [14] where the key is to use mixed input states. In Ref. [31], a LGI test using two-time NSIT measurements is called an intermediate test of macrorealism.

\section{APPENDIX P: LGI AND SIT AS EFFICIENT QUANTUM WITNESSES}

This work shows the violation of a LGI using a mechanical oscillator, a system that allows us to explore the quantum-to-classical transition in a natural way. Further, we also explore SIT as an alternative quantum witness. Both methods require few measurements for the confirmation of the quantum states: LGI needs 12 fluorescence detections, while SIT needs 6. This amount is much less than we typically require to extract a negative Wigner function point. For the latter, we extract the Fock state populations of the oscillator from a sideband flopping curve, which requires around 200 fluorescence detections [24]. We find that the LGI methods require excellent frequency stability of the mechanical oscillator under test. The SIT quantum witness has the advantage of involving only measurements at two times. However, SIT needs $m_{\alpha_{B}} \neq 0$, which requires more-involved oscillator input states.

\section{APPENDIX Q: SIT MEASUREMENTS VIOLATING A LEGGETT-GARG INEQUALITY}

Here, we give an explicit procedure for how to violate the Leggett-Garg inequality, $L=C_{A B}+C_{B C}-C_{A C} \leq 1$, having observed SIT between two modular measurements on an input state. This procedure is equivalent to the one used and discussed in Ref. [34] and is briefly commented on in Ref. [16].

We consider two measurements, $B$ and $C$, which we read out by coupling them to an ancilla qubit. Thus, the measurement of the qubit has two possible outcomes, up and down, which we label $U$ and $D$. The only assumption we make about the measurements $B$ and $C$ is that $B$ is SIT to $C$ when the input state $|\psi\rangle$ is measured. This means $P_{C}(c) \neq \sum_{b} P_{C B}(c, b)=P_{C(B)}(c)$. Thus, one of the two probabilities needs to be bigger than the other. Without loss of generality, we choose $P_{C}(D)<P_{C(B)}(D)$, and we define $a \equiv P_{C(B)}(D)-P_{C}(D)$ to be the difference between the two. [In the case of $P_{C}(U)>P_{C(B)}(U)$, we can modify the protocol slightly.] Measurement $A$ is simply the state preparation or confirmation of the state preparation of $|\psi\rangle$. The key point of the protocol is to assign different measurement results to the outcomes $U, D$ in each of the measurements $A, B, C$. The assigned results are always $r= \pm 1$, which is compatible with the assumption $|r| \leq 1$ used in the proof of the LGI [16]. To be more specific, the three measurements violating the LGI are given as follows:

$t_{0}$ : Initial state preparation of $|\uparrow\rangle|\psi\rangle$.

$t_{1}$ : Measurement $A$ : Readout of the qubit. We assign the value +1 to $U$ and -1 to $D$; $f_{A}(U)=+1, f_{A}(D)=-1$.

$t_{2}$ : Measurement $B$ and both results $D$ and $U$ are identified with $+1 ; f_{B}(D)=f_{B}(U)=+1$.

$t_{3}$ : Measurement $C$. We assign the value -1 to result $U$ and +1 to result $D ; f_{C}(U)=-1, f_{C}(D)=1$.

Note that the assignment of a constant value for measurement $B$ can be interpreted as performing the measurement but not looking at the result. We can now calculate the violation of the LGI. Here, $C_{A B}=1$ since measurement $A$ always gives an up result, and in measurement $B$, we assigned the constant value of +1 . The correlator $C_{A C}=\sum_{c} f_{C}(c) P_{C}(c)$ simplifies to the expectation value of measurement $C$ since measurement 

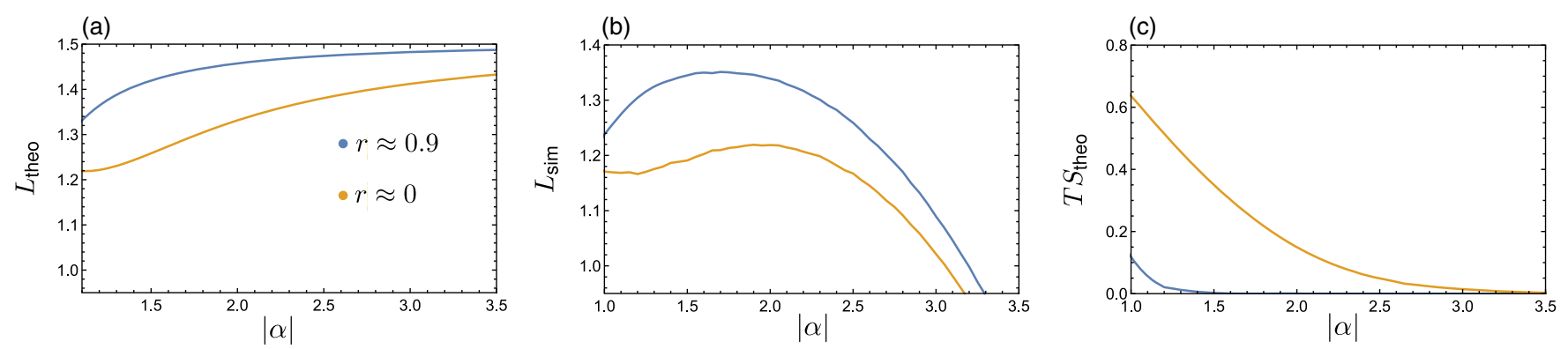

FIG. 12. Theoretical comparison of a LGI experiment using a ground-state cooled or a squeezed oscillator state. Results are presented for a squeezing parameter $r \approx 0.9$. (a) Analytic calculation of the achievable $L$ values. (b) Simulated $L$ values including the same motional dephasing and linewidths as in the simulations in the main part of the paper Fig. 4(b). (c) Analytic calculation of the amount of $T S$ present during the measurements.

$A$ is only confirming the state preparation. Note that $C_{B C}=\sum_{c} f_{C}(c) \times \sum_{b} P_{C B}(c, b)$ simplifies since we assigned in measurement $B$ the constant value of +1 . Thus,

$$
\begin{aligned}
L & =1+\left(\sum_{c} f_{C}(c) \sum_{b} P_{C B}(c, b)-\sum_{c} f_{C}(c) P_{C}(c)\right) \\
& =1+\sum_{c} f_{C}(c)\left(\sum_{b} P_{C B}(c, b)-P_{C}(c)\right) \\
& =1+2 a>1 .
\end{aligned}
$$

We can also see that, in the case of $P_{C}(D)>P_{C(B)}(D)$, we can change the assignment of results in measurement $C$ to $f_{C}(U)=1, f_{C}(D)=-1$.

\section{APPENDIX R: GKP STATE ALLOWING $S=1$}

This result can be seen in various ways. Let us consider the formula for $S$ in the symmetric implementation: $S=\frac{1}{2}(1-\cos (\Phi))\left|m_{\alpha_{B}}\right| \cos \left(\arg \left(m_{\alpha_{B}}\right)\right)$. Here, $S=1$ requires $\Phi=\pi$ and $m_{\alpha_{B}}=1$. This is fulfilled if we choose a GKP [10] input state: $\sum_{l=-\infty}^{\infty} \hat{\mathcal{D}}\left(l \alpha_{B}\right)|x=0\rangle$, with $\alpha_{B} \in$ $\mathbb{R}$ and $\alpha_{A}=i \pi / \alpha_{B}$.

\section{APPENDIX S: POTENTIAL IMPROVEMENT OF LGI VIOLATION USING SQUEEZED OSCILLATOR INPUT STATES}

The violation of the LGI gains two advantages from the use of squeezed input states: an increased violation and a reduction in SIT for a given displacement. We maximize the $L$ value for squeezed initial oscillator states $\hat{S}(\xi)|0\rangle$, with $\hat{S}(\xi)=e^{\left(\xi^{*} \hat{a}^{2}-\xi \hat{a}^{\hat{I}^{2}}\right) / 2}, \xi=r e^{i \phi}$ the squeezing operator and parameter. Comparing a squeezed state with $r \approx 0.9$ to a ground state, we find that with the same displacement, the squeezed state allows higher violation [see Fig. 12(a)]. The simulation of realistic dephasing noise [Fig. 12(b)] shows that this advantage is still present in a realistic scenario. The SIT built into the measurements also drops much quicker for the squeezed state compared to the ground state. Analytic calculations of the built-in SIT are shown in Fig. 12(c). Furthermore, the created states for a fixed $|\alpha|$ are, in some sense, more macroscopic since the ratio of separation to relevant wave-packet extent (approximately the squeezed wave-packet size) is larger. For $r=0.9$, the ratio is improved by a factor of approximately 2.5 .

[1] M. Ozawa, Universally Valid Reformulation of the Heisenberg Uncertainty Principle on Noise and Disturbance in Measurement, Phys. Rev. A 67, 042105 (2003).

[2] M. Ozawa, Uncertainty Relations for Joint Measurements of Noncommuting Observables, Phys. Lett. A 320, 367 (2004).

[3] M. J. W. Hall, Prior Information: How to Circumvent the Standard Joint-Measurement Uncertainty Relation, Phys. Rev. A 69, 052113 (2004).

[4] K. Duivenvoorden, B. M. Terhal, and D. Weigand, SingleMode Displacement Sensor, Phys. Rev. A 95, 012305 (2017).

[5] Y. Aharonov and D. Rohrlich, Modular Variables, in Quantum Paradoxes (Wiley-VCH Verlag, Weinheim, 2008), pp. 61-75.

[6] Y. Aharonov, H. Pendleton, and A. Petersen, Modular Variables in Quantum Theory, Int. J. Theor. Phys. 2, 213 (1969).

[7] S. Popescu, Dynamical Quantum Non-locality, Nat. Phys. 6, 151 (2010).

[8] A. Asadian, Č. Brukner, and P. Rabl, Probing Macroscopic Realism via Ramsey Correlation Measurements, Phys. Rev. Lett. 112, 190402 (2014).

[9] A. Asadian, C. Budroni, F. E. S. Steinhoff, P. Rabl, and O. Gühne, Contextuality in Phase Space, Phys. Rev. Lett. 114, 250403 (2015).

[10] D. Gottesman, A. Kitaev, and J. Preskill, Encoding a Qubit in an Oscillator, Phys. Rev. A 64, 012310 (2001).

[11] V. V. Albert, K. Noh, K. Duivenvoorden, R. T. Brierley, P. Reinhold, C. Vuillot, L. Li, C. Shen, S. M. Girvin, 
B. M. Terhal, and L. Jiang, Performance and Structure of Bosonic Codes, arXiv:1708.05010.

[12] B. M. Terhal and D. Weigand, Encoding a Qubit into a Cavity Mode in Circuit QED Using Phase Estimation, Phys. Rev. A 93, 012315 (2016).

[13] B. C. Travaglione and G. J. Milburn, Preparing Encoded States in an Oscillator, Phys. Rev. A 66, 052322 (2002).

[14] J. Kofler and Č. Brukner, Condition for Macroscopic Realism Beyond the Leggett-Garg Inequalities, Phys. Rev. A 87, 052115 (2013).

[15] C.-M. Li, N. Lambert, Y.-N. Chen, G.-Y. Chen, and F. Nori, Witnessing Quantum Coherence: From Solid-State to Biological Systems, Sci. Rep. 2, 885 (2012).

[16] C. Emary, N. Lambert, and F. Nori, Leggett-Garg Inequalities, Rep. Prog. Phys. 77, 016001 (2014).

[17] A. J. Leggett and A. Garg, Quantum Mechanics Versus Macroscopic Realism: Is the Flux There When Nobody Looks?, Phys. Rev. Lett. 54, 857 (1985).

[18] D. J. Wineland, C. R. Monroe, W. M. Itano, D. Leibfried, B. King, and D. M. Meekhof, Experimental Issues in Coherent Quantum-State Manipulation of Trapped Atomic Ions, J. Res. Natl. Inst. Stand. Technol. 103, 259 (1998).

[19] H. M. Wiseman and G. J. Milburn, Quantum Measurement and Control (Cambridge University Press, Cambridge, England, 2009).

[20] S. J. Devitt, W. J. Munro, and K. Nemoto, Quantum Error Correction for Beginners, Rep. Prog. Phys. 76, 076001 (2013).

[21] W. P. Schleich, WKB and Berry Phase, in Quantum Optics in Phase Space (Wiley-VCH Verlag, Weinheim, 2005), pp. 171-188.

[22] B.-G. Englert, K. L. Lee, A. Mann, and M. Revzen, Periodic and Discrete Zak Bases, J. Phys. A 39, 1669 (2006).

[23] P. C. Haljan, K.-A. Brickman, L. Deslauriers, P. J. Lee, and C. Monroe, Spin-Dependent Forces on Trapped Ions for Phase-Stable Quantum Gates and Entangled States of Spin and Motion, Phys. Rev. Lett. 94, 153602 (2005).

[24] D. Kienzler, C. Flühmann, V. Negnevitsky, H.-Y. Lo, M. Marinelli, D. Nadlinger, and J. P. Home, Observation of Quantum Interference between Separated Mechanical Oscillator Wave Packets, Phys. Rev. Lett. 116, 140402 (2016).
[25] H.-Y. Lo, D. Kienzler, L. de Clercq, M. Marinelli, V. Negnevitsky, B. Keitch, and J.P. Home, Spin-Motion Entanglement and State Diagnosis with Squeezed Oscillator Wavepackets, Nature (London) 521, 336 (2015).

[26] S. Kochen and E. P. Specker, The Problem of Hidden Variables in Quantum Mechanics, Indiana Univ. Math. J. 17, 59 (1968).

[27] B. Hensen, H. Bernien, A. E. Dreau, A. Reiserer, N. Kalb, M. S. Blok, J. Ruitenberg, R. F. L. Vermeulen, R. N. Schouten, C. Abellan, W. Amaya, V. Pruneri, M. W. Mitchell, M. Markham, D. J. Twitchen, D. Elkouss, S. Wehner, T. H. Taminiau, and R. Hanson, Loophole-Free Bell Inequality Violation Using Electron Spins Separated by 1.3 Kilometres, Nature (London) 526, 682 (2015).

[28] C. Monroe, D. M. Meekhof, B. E. King, and D. J. Wineland, A "Schrödinger Cat" Superposition State of an Atom, Science 272, 1131 (1996).

[29] K. G. Johnson, J. D. Wong-Campos, B. Neyenhuis, J. Mizrahi, and C. Monroe, Ultrafast Creation of Large Schrödinger Cat States of an Atom, Nat. Commun. 8, 697 (2017).

[30] D. Kienzler, H.-Y. Lo, V. Negnevitsky, C. Flühmann, M. Marinelli, and J. P. Home, Quantum Harmonic Oscillator State Control in a Squeezed Fock Basis, Phys. Rev. Lett. 119, 033602 (2017).

[31] J. J. Halliwell, Comparing Conditions for Macrorealism: Leggett-Garg Inequalities vs No-Signaling in Time, Phys. Rev. A 96, 012121 (2017).

[32] J. V. Kujala, E. N. Dzhafarov, and J.-Å. Larsson, Necessary and Sufficient Conditions for an Extended Noncontextuality in a Broad Class of Quantum Mechanical Systems, Phys. Rev. Lett. 115, 150401 (2015).

[33] A. Ketterer, Ph.D. thesis, Physique en Ile-de-France, 2016.

[34] C. Robens, W. Alt, D. Meschede, C. Emary, and A. Alberti, Ideal Negative Measurements in Quantum Walks Disprove Theories Based on Classical Trajectories, Phys. Rev. X 5 , 011003 (2015).

[35] T. C. Ralph, S. D. Bartlett, J. L. O'Brien, G. J. Pryde, and H. M. Wiseman, Quantum Nondemolition Measurements for Quantum Information, Phys. Rev. A 73, 012113 (2006). 